University of Nebraska - Lincoln

DigitalCommons@University of Nebraska - Lincoln

US Department of Energy Publications

U.S. Department of Energy

2007

\title{
Peridynamic States and Constitutive Modeling
}

S. A. Silling

Sandia National Laboratories, sasilli@sandia.gov

M. Epton

The Boeing Company

O. Weckner

The Boeing Company

J. Xu

The Boeing Company

E. Askari

The Boeing Company

Follow this and additional works at: https://digitalcommons.unl.edu/usdoepub

Part of the Bioresource and Agricultural Engineering Commons

Silling, S. A.; Epton, M.; Weckner, O.; Xu, J.; and Askari, E., "Peridynamic States and Constitutive Modeling" (2007). US Department of Energy Publications. 95.

https://digitalcommons.unl.edu/usdoepub/95

This Article is brought to you for free and open access by the U.S. Department of Energy at DigitalCommons@University of Nebraska - Lincoln. It has been accepted for inclusion in US Department of Energy Publications by an authorized administrator of DigitalCommons@University of Nebraska - Lincoln. 


\title{
Peridynamic States and Constitutive Modeling
}

\author{
S. A. Silling • M. Epton • O. Weckner · J. Xu • E. Askari
}

Received: 14 March 2007 / Accepted: 12 June 2007 /

Published online: 14 July 2007

(C) Springer Science + Business Media B.V. 2007

\begin{abstract}
A generalization of the original peridynamic framework for solid mechanics is proposed. This generalization permits the response of a material at a point to depend collectively on the deformation of all bonds connected to the point. This extends the types of material response that can be reproduced by peridynamic theory to include an explicit dependence on such collectively determined quantities as volume change or shear angle. To accomplish this generalization, a mathematical object called a deformation state is defined, a function that maps any bond onto its image under the deformation. A similar object called a force state is defined, which contains the forces within bonds of all lengths and orientation. The relation between the deformation state and force state is the constitutive model for the material. In addition to providing a more general capability for reproducing material response, the new framework provides a means to incorporate a constitutive model from the conventional theory of solid mechanics directly into a peridynamic model. It also allows the condition of plastic incompressibility to be enforced in a peridynamic material model for permanent deformation analogous to conventional plasticity theory.
\end{abstract}

Keywords Peridynamic $\cdot$ Material model Constitutive model • Nonlocal elasticity • Fracture $\cdot$ Plastic flow

Mathematics Subject Classifications (2000) $74 \mathrm{~A} 05 \cdot 74 \mathrm{~A} 20$

S. A. Silling $(\varangle)$

Multiscale Dynamic Material Modeling Department,

Sandia National Laboratories,

Albuquerque, New Mexico, USA

e-mail: sasilli@sandia.gov

M. Epton · O. Weckner · J. Xu · E. Askari

Math Group, The Boeing Company, Bellevue, Washington, USA 


\section{Introduction}

An alternative formulation for continuum mechanics, called the peridynamic model [1], was proposed several years ago. This model offered the ability to apply its basic equations directly on a surface of discontinuity, such as a crack. This is in contrast to the classical theory, in which the partial differential equations fail to be applicable directly on such a singularity. Because of its nonlocal nature and its similarity to molecular dynamics, the peridynamic model also provides a natural setting in which to model long-range forces, such as van der Waals forces, that are important in nanoscale applications [2].

The original form of the peridynamic model, which for purposes of this paper will be called the bond-based theory, uses a two-particle force function $\mathbf{f}$ to describe the interaction between material particles:

$$
\rho(\mathbf{x}) \ddot{\mathbf{u}}(\mathbf{x}, t)=\int_{\mathcal{H}_{\mathbf{x}}} \mathbf{f}\left(\mathbf{u}\left(\mathbf{x}^{\prime}, t\right)-\mathbf{u}(\mathbf{x}, t), \mathbf{x}^{\prime}-\mathbf{x}\right) d V_{\mathbf{x}^{\prime}}+\mathbf{b}(\mathbf{x}, t),
$$

where $\mathcal{H}_{\mathbf{x}}$ is a neighborhood of $\mathbf{x}, \mathbf{u}$ is the displacement vector field, $\mathbf{b}$ is a prescribed body force density field, $\rho$ is mass density in the reference configuration, and $\mathbf{f}$ is a pairwise force function whose value is the force vector (per unit volume squared) that the particle $\mathbf{x}^{\prime}$ exerts on the particle $\mathbf{x}$. In the following discussion, the relative position of these two particles in the reference configuration will be denoted by $\xi$ and their relative displacement by $\eta$ :

$$
\xi=\mathbf{x}^{\prime}-\mathbf{x}, \quad \eta=\mathbf{u}\left(\mathbf{x}^{\prime}, t\right)-\mathbf{u}(\mathbf{x}, t) .
$$

Using these definitions, $\boldsymbol{\eta}+\boldsymbol{\xi}$ represents the current relative position vector between the particles. The vector $\xi$ is called a bond. The physical nature of the interaction between $\mathbf{x}$ and $\mathbf{x}^{\prime}$ need not be specified. The concept of a bond that extends over a finite distance is a fundamental difference between the peridynamic theory and the classical theory, which is based on the idea of contact forces (interactions between particles that are in direct contact with each other). Certain restrictions on $\mathbf{f}$ arise from basic mechanical considerations [1]:

$$
\mathbf{f}(-\eta,-\xi)=-\mathbf{f}(\boldsymbol{\eta}, \boldsymbol{\xi}), \quad(\xi+\eta) \times \mathbf{f}(\boldsymbol{\eta}, \boldsymbol{\xi})=\mathbf{0} \quad \forall \boldsymbol{\eta}, \boldsymbol{\xi}
$$

It is convenient to assume that for a given material, there is a positive number $\delta$, called the horizon, such that

$$
|\boldsymbol{\xi}|>\delta \quad \Longrightarrow \quad \mathbf{f}(\boldsymbol{\eta}, \boldsymbol{\xi})=\mathbf{0} \quad \forall \boldsymbol{\eta}
$$

For the remainder of this discussion, $\mathcal{H}_{\mathbf{x}}$ will denote the spherical neighborhood of $\mathbf{x}$ with radius $\delta$.

The bond-based peridynamic model is similar to models proposed by Kunin [3] and by Rogula [4] in the investigation of continuum properties of crystals based on interatomic interactions. Recent theoretical work on the peridynamic model includes the study of discontinuities and other mathematical aspects of deformations in one-dimensional constitutively linear bodies [5, 6]. Application of the method to martensitic phase transformations has demonstrated that it determines, without the use of a supplemental kinetic relation, the dependence of phase boundary velocity on the conditions near the phase boundary [7]. Practical applications include damage and failure in reinforced concrete under quasi-static loading $[8,9,10]$, and 
impact against brittle structures [11]. The method has also been applied to analysis of damage in a composite laminate under impact loading [12], and to failure of brazed joints [13]. The effect of long-range forces on the deformation and failure of nanofiber membranes was investigated in [14]. Numerical techniques for solving the peridynamic equations include the EMU code $[15,16]$, as well as alternative quadrature techniques and solution methods [9, 10, 17].

The present paper deals with a generalization of the bond-based theory just described. In the bond-based theory, the pairwise force function $\mathbf{f}$ contains all the constitutive information about the material. At least three difficulties occur with this approach:

- In many cases it is an oversimplification to assume that any pair of particles interacts only through a central potential that is totally independent of all other local conditions. For example, this assumption results (for an isotropic, linear, microelastic material) in an effective Poisson ratio of $1 / 4$.

- Because historically in continuum mechanics the constitutive behavior of a material has been described in terms of a stress tensor, the requirement to completely recast a material model in terms of a pairwise force function becomes a practical barrier to use of the peridynamic approach.

- Although plasticity can be included in the bond-based theory by permitting permanent deformation of individual bonds, this results in permanent deformation of a material undergoing a volumetric strain (without shear). While this feature could be appropriate for modeling a porous material, it is inconsistent with plastic incompressibility in metals, i.e., the experimental observation that only shear deformations can involve plastic response.

The first of these issues can be addressed by introducing rotational degrees of freedom into a bond-based peridynamic model [10]. This allows materials with a Poisson ratio other than $1 / 4$ to be represented. However, although this is a big improvement over the original theory, it is not clear that this enhancement can successfully address the second and third issues.

The methods described in the present paper attempt to address all these issues by rewriting the material-dependent part of the peridynamic model, introducing a mathematical object called a force state that is in some ways similar to the traditional stress tensor of classical continuum mechanics. It is shown that by using this concept, the basic peridynamic theory can be generalized to include materials with any Poisson ratio. Also, because of the similarity to stress tensors, it is possible to apply constitutive models in the classical theory more or less directly in the peridynamic theory. This generalized version of the peridynamic theory to be described in the remainder of this paper will be called the state-based theory, as opposed to the bondbased theory already described.

Sections 2, 3, and 4 of this paper provide some mathematical preliminaries on peridynamic states. Section 5 defines a notion of differentiation of functions of states. Dependence of states on position in a body is considered in Section 6, which sets the stage for the equation of motion in the state based theory in Section 7. Some general features of peridynamic constitutive modeling are discussed in Section 8. Mathematical conditions for isotropy and objectivity are introduced in Section 9, and Sections 10 and 11 discuss elastic material models. In Section 12 the idea of correspondence is introduced, which defines what it means to require a peridynamic material model to 
agree with a given classical material model for a given deformation. In Sections 13, 14 , and 15, separation of peridynamic material models into dilatational and deviatoric responses is discussed, allowing linear fluid and solid elastic models to be defined. A way of modeling elastic-plastic response is discussed in Section 16, using an idea of a yield surface analogous to the classical theory, and allowing the condition of plastic incompressibility to be enforced. An example of a material in which the strain energy depends explicitly on changes of relative angle between peridynamic bods is given in Section 17. Section 18 derives a general procedure for incorporating a material model from the classical theory into the peridynamic framework.

\section{States}

Let $\delta$ be a positive number, and let $\mathcal{H}$ be a spherical neighborhood of radius $\delta$ centered at the origin in $\mathbb{R}^{3}$. Let $\mathcal{L}_{m}$ denote the set of all tensors of order $m$ (thus $\left.\mathcal{L}_{0}=\mathbb{R}\right)$.

Definition 2.1 A state of order $m$ is a function $\underline{\mathbf{A}}\langle\cdot\rangle: \mathcal{H} \rightarrow \mathcal{L}_{m}$.

Thus, the image of a vector $\boldsymbol{\xi} \in \mathcal{H}$ under the state $\underline{\mathbf{A}}$ is a tensor of order $m$, written $\underline{\mathbf{A}}\langle\boldsymbol{\xi}\rangle$. The set of all states of order $m$ is denoted $\mathcal{A}_{m}$. Angle brackets are used to indicate the vector on which a state operates.

The majority of states to be considered in this paper fall into either of two categories, so abbreviated notations will be introduced for these:

- A state of order 1 is called a vector state. The set of all vector states is denoted $\mathcal{V}$, thus $\mathcal{V}=\mathcal{A}_{1}$. Vector states and other states of order $m \geq 1$ are usually written in uppercase bold font with an underscore, e.g., $\underline{\mathbf{A}}$.

- A state of order 0 is called a scalar state. The set of all scalar states is denoted $\mathcal{S}$, thus $\mathcal{S}=\mathcal{A}_{0}$. Scalar states are usually written as lower case, non-bold font with an underscore, e.g., $\underline{a}$.

If components are used in a Cartesian coordinate system, an order $m$ state has $m$ components that are written as $A_{i_{1} i_{2} \ldots i_{m}}$. The vectors in $\mathcal{H}$ on which a state operates are written in angle brackets $\langle\cdot\rangle$ to distinguish them from other quantities that the state itself may depend on.

Definition 2.2 Let $\underline{\mathbf{A}} \in \mathcal{A}_{m}$ and $\underline{\mathbf{B}} \in \mathcal{A}_{m}$. The sum of the states $\underline{\mathbf{A}}$ and $\underline{\mathbf{B}}$ is a state in $\mathcal{A}_{m}$ defined by

$$
(\underline{\mathbf{A}}+\underline{\mathbf{B}})\langle\boldsymbol{\xi}\rangle=\underline{\mathbf{A}}\langle\boldsymbol{\xi}\rangle+\underline{\mathbf{B}}\langle\boldsymbol{\xi}\rangle \quad \forall \xi \in \mathcal{H} .
$$

The difference $\underline{\mathbf{A}}-\underline{\mathbf{B}}$ is also defined in the obvious way.

Definition 2.3 Let $\underline{\mathbf{A}} \in \mathcal{A}_{m}$ and $\underline{\mathbf{V}} \in \mathcal{V}$. The composition of states $\underline{\mathbf{A}}$ and $\underline{\mathbf{V}}$ is a state in $\mathcal{A}_{m}$ defined by

$$
(\underline{\mathbf{A}} \circ \underline{\mathbf{V}})\langle\boldsymbol{\xi}\rangle=\underline{\mathbf{A}}\langle\underline{\mathbf{V}}\langle\boldsymbol{\xi}\rangle\rangle \quad \forall \boldsymbol{\xi} \in \mathcal{H} .
$$


Definition 2.4 The point product of two states $\underline{\mathbf{A}} \in \mathcal{A}_{m+p}$ and $\underline{\mathbf{B}} \in \mathcal{A}_{p}$ is a state in $\mathcal{A}_{m}$ defined by

$$
(\underline{\mathbf{A B}})_{i_{1} \ldots i_{m}}\langle\boldsymbol{\xi}\rangle=\underline{A}_{i_{1} \ldots i_{m} j_{1} \ldots j_{p}}\langle\boldsymbol{\xi}\rangle \underline{B}_{j_{1} \ldots j_{p}}\langle\boldsymbol{\xi}\rangle \quad \forall \boldsymbol{\xi} \in \mathcal{H}
$$

Similarly,

$$
(\underline{\mathbf{B A}})_{i_{1}, \ldots, i_{m}}\langle\boldsymbol{\xi}\rangle=\underline{B}_{j_{1}, \ldots, j_{p}}\langle\boldsymbol{\xi}\rangle \underline{A}_{j_{1}, \ldots, j_{p}, i_{1}, \ldots, i_{m}}\langle\boldsymbol{\xi}\rangle \quad \forall \boldsymbol{\xi} \in \mathcal{H}
$$

Note that summation occurs over all the innermost indices. If either or both of the states is a scalar state, then the point product is commutative: $\underline{\mathbf{A}} b=\underline{b \mathbf{A}}$. The point product is also commutative if $m=0$, i.e., if the two states are of the same order.

Definition 2.5 The reference position vector state $\underline{\mathbf{X}} \in \mathcal{V}$ and the identity scalar state $\underline{1} \in \mathcal{S}$ are defined by

$$
\underline{\mathbf{X}}\langle\xi\rangle=\xi, \quad \underline{1}\langle\xi\rangle=\left\{\begin{array}{ll}
1 & \text { if } \boldsymbol{\xi} \neq \mathbf{0} \\
0 & \text { if } \boldsymbol{\xi}=\mathbf{0}
\end{array} \quad \forall \xi .\right.
$$

The null vector state $\underline{\mathbf{0}} \in \mathcal{V}$ and null scalar state $\underline{0} \in \mathcal{S}$ are also defined in the obvious way,

$$
\underline{\mathbf{0}}\langle\boldsymbol{\xi}\rangle=\mathbf{0}, \quad \underline{0}\langle\boldsymbol{\xi}\rangle=0 \quad \forall \boldsymbol{\xi} .
$$

Definition 2.6 The dot product of two states $\underline{\mathbf{A}}$ and $\underline{\mathbf{B}}$ is defined by

$$
\underline{\mathbf{A}} \cdot \underline{\mathbf{B}}=\int_{\mathcal{H}}(\underline{\mathbf{A B}})\langle\boldsymbol{\xi}\rangle d V_{\xi}
$$

(The integrand is a point product, defined above.) If $\underline{\mathbf{A}}$ and $\underline{\mathbf{B}}$ are of the same order, or if either of them is a scalar state, then $\underline{\mathbf{A}} \bullet \underline{\mathbf{B}}=\underline{\mathbf{B}} \bullet \underline{\mathbf{A}}$.

Definition 2.7 If $\underline{\mathbf{A}} \in \mathcal{A}_{m}$, then the magnitude state of $\underline{\mathbf{A}}$ is the scalar state defined by

$$
|\underline{\mathbf{A}}|\langle\boldsymbol{\xi}\rangle=\sqrt{(\underline{\mathbf{A A}})\langle\xi\rangle} \quad \forall \xi \in \mathcal{H} .
$$

(For any $\xi$, the point product under the radical sign is a non-negative scalar.)

Definition 2.8 If $\underline{\mathbf{N}}$ is a state, and if $|\underline{\mathbf{N}}|=\underline{1}$, then $\underline{\mathbf{N}}$ is a unit state.

Definition 2.9 The norm of a state $\underline{\mathbf{A}}$ is a scalar defined by

$$
\|\underline{\mathbf{A}}\|=\sqrt{\underline{\mathbf{A}} \bullet \underline{\mathbf{A}}}
$$

Definition 2.10 Define the direction of a state $\underline{\mathbf{A}} \in \mathcal{A}_{m}$ to be the state Dir $\underline{\mathbf{A}} \in \mathcal{A}_{m}$ given by

$$
(\operatorname{Dir} \underline{\mathbf{A}})\langle\boldsymbol{\xi}\rangle= \begin{cases}\mathbf{0} & \text { if }|\underline{\mathbf{A}}|\langle\boldsymbol{\xi}\rangle=0, \quad \forall \boldsymbol{\xi} \in \mathcal{H} . \\ \underline{\mathbf{A}}\langle\boldsymbol{\xi}\rangle /|\underline{\mathbf{A}}|\langle\boldsymbol{\xi}\rangle & \text { otherwise }\end{cases}
$$




\section{Vector States and Tensors}

It is readily verified that for any order $m, \mathcal{A}_{m}$ is a real Euclidean linear space [18] with a scalar product supplied by the dot product defined in (11). This is in spite of the fact that the dependence of a state on the operand $\xi$ may be nonlinear.

The concept of a vector state is similar to that of a second order tensor in that they both map vectors into vectors, but with three important differences:

- A state is not in general a linear function of $\xi$.

- A state is not in general a continuous function of $\xi$.

- The real Euclidean space $\mathcal{V}$ is infinite-dimensional, while the real Euclidean space $\mathcal{L}_{2}$ (the set of second order tensors) has dimension 9.

These differences are illustrated schematically in Fig. 1. From the above list, it is clear that vector states are more general than second order tensors, and that second order tensors are in some sense a special case of vector states. To make this idea more precise, the following tools called "expansion" and "reduction" are now introduced to translate between the two concepts.

Definition 3.1 Given a second order tensor $\mathbf{W}$, let $\underline{\mathcal{E}}(\mathbf{W}) \in \mathcal{V}$ be the vector state expanded from $\mathbf{W}$, defined by

$$
\underline{\mathcal{E}}(\mathbf{W})\langle\xi\rangle=\mathbf{W} \xi \quad \forall \xi .
$$

Definition 3.2 Let a scalar state $\underline{\omega} \in \mathcal{S}$ be given. Suppose that $\underline{\omega}$ is nonnegative on $\mathcal{H}$. Suppose further that there is a subregion with nonzero volume $\mathcal{H}^{\prime} \subset \mathcal{H}$ such that $\underline{\omega}$ is strictly positive on $\mathcal{H}^{\prime}$. Then $\underline{\omega}$ is called an influence function. If $\underline{\omega}$ is an influence

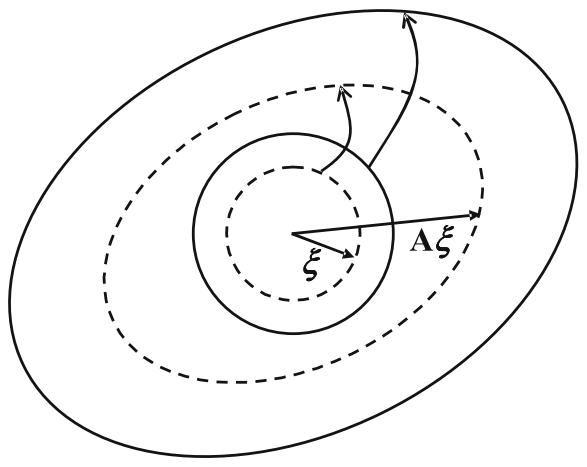

Symmetric tensor $\mathbf{A}$

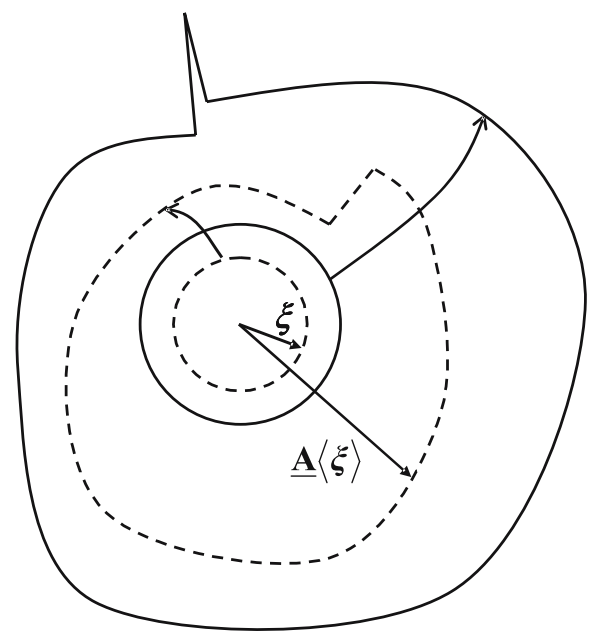

Vector state $\underline{\mathbf{A}}$

Fig. 1 A symmetric second order tensor maps a sphere into an ellipsoid, while a state maps it into a more complex and possibly discontinuous surface 
function and $\underline{\omega}\langle\boldsymbol{\xi}\rangle$ depends only on the scalar $|\boldsymbol{\xi}|$, then $\underline{\omega}$ is said to be spherical, and we write $\underline{\omega}\langle\boldsymbol{\xi}\rangle=\underline{\omega}_{s}\langle|\boldsymbol{\xi}|\rangle$.

Let the dyadic product of two vectors $\mathbf{a}$ and $\mathbf{b}$ be denoted $\mathbf{C}=\mathbf{a} \otimes \mathbf{b}$. (In rectangular coordinates, $C_{i j}=a_{i} b_{j}$.)

Definition 3.3 Let an influence function $\underline{\omega} \in \mathcal{S}$ be given. For any two vector states $\underline{\mathbf{A}} \in \mathcal{V}$ and $\underline{\mathbf{B}} \in \mathcal{V}$, let $\underline{\mathbf{A}} * \underline{\mathbf{B}}$ be the second order tensor defined by

$$
\underline{\mathbf{A}} * \underline{\mathbf{B}}=\int_{\mathcal{H}} \underline{\omega}\langle\boldsymbol{\xi}\rangle \underline{\mathbf{A}}\langle\boldsymbol{\xi}\rangle \otimes \underline{\mathbf{B}}\langle\boldsymbol{\xi}\rangle d V_{\boldsymbol{\xi}}
$$

The tensor $\underline{\mathbf{A}} * \underline{\mathbf{B}}$ is called the tensor product of $\underline{\mathbf{A}}$ and $\underline{\mathbf{B}}$.

Definition 3.4 Let an influence function $\underline{\omega} \in \mathcal{S}$ be given. Define the shape tensor K by

$$
\mathbf{K}=\underline{\mathbf{X}} * \underline{\mathbf{X}}
$$

where $\underline{\mathbf{X}} \in \mathcal{V}$ is defined in (9). Note that $\mathbf{K}$ is symmetric.

Lemma 3.1 Let an influence function $\underline{\omega} \in \mathcal{S}$ be given. Then $\mathbf{K}$ defined in (17) is positive definite.

Proof Since $\mathbf{K}$ is symmetric, it has three real eigenvalues, not necessarily distinct. Let $\lambda$ be an eigenvalue of $\mathbf{K}$, and let $\mathbf{a}$ be a unit eigenvector associated with $\lambda$. Then

$$
\begin{aligned}
\lambda & =\mathbf{a} \cdot \mathbf{K a}=\mathbf{a} \cdot\left(\int_{\mathcal{H}} \underline{\omega}\langle\boldsymbol{\xi}\rangle \boldsymbol{\xi} \otimes \boldsymbol{\xi} d V_{\boldsymbol{\xi}}\right) \mathbf{a} \\
& =\int_{\mathcal{H}} \underline{\omega}\langle\boldsymbol{\xi}\rangle(\mathbf{a} \cdot \boldsymbol{\xi})^{2} d V_{\boldsymbol{\xi}} \geq \int_{\mathcal{H}^{\prime}} \underline{\omega}\langle\boldsymbol{\xi}\rangle(\mathbf{a} \cdot \boldsymbol{\xi})^{2} d V_{\boldsymbol{\xi}}>0
\end{aligned}
$$

in which the last step follows from the definition of $\underline{\omega}$. (Recall that $\underline{\omega} \geq 0$ on $\mathcal{H}$ and $\underline{\omega}>0$ on $\mathcal{H}^{\prime} \subset \mathcal{H}$.) Therefore, all the eigenvalues of $\mathbf{K}$ are positive, so the tensor is positive definite.

Definition 3.5 Let an influence function $\underline{\omega} \in \mathcal{S}$ be given, and let $\underline{\mathbf{A}} \in \mathcal{V}$ be a vector state. Define a second order tensor $\mathcal{R}\{\underline{\mathbf{A}}\}$ by

$$
\mathcal{R}\{\underline{\mathbf{A}}\}=(\underline{\mathbf{A}} * \underline{\mathbf{X}}) \mathbf{K}^{-1} .
$$

$\mathcal{R}\{\underline{\mathbf{A}}\}$ is called the tensor reduced from $\underline{\mathbf{A}}$. (The existence of $\mathbf{K}^{-1}$ follows from the lemma above.) 
Example Let $\mathbf{Z}$ be a given tensor, and let $\underline{\mathbf{Z}}$ be the vector state expanded from $\mathbf{Z}$, i.e., $\underline{\mathbf{Z}}=\underline{\mathcal{E}}\{\mathbf{Z}\}$. Using the component forms of (15), (16), and (19), $\mathcal{R}\{\underline{\mathbf{Z}}\}$ is computed as follows:

$$
\begin{aligned}
\mathcal{R}_{i j}\{\underline{\mathbf{Z}}\} & =(\underline{\mathbf{Z}} * \underline{\mathbf{X}})_{i k} K_{k j}^{-1} \\
& =\left(\int_{\mathcal{H}} \underline{\omega}\langle\xi\rangle\left(Z_{i p} \xi_{p}\right) \xi_{k} d V_{\boldsymbol{\xi}}\right) K_{k j}^{-1} \\
& =Z_{i p}\left(\int_{\mathcal{H}} \underline{\omega}\langle\boldsymbol{\xi}\rangle \xi_{p} \xi_{k} d V_{\boldsymbol{\xi}}\right) K_{k j}^{-1} \\
& =Z_{i p} K_{p k} K_{k j}^{-1} \\
& =Z_{i p} \delta_{p j} \\
& =Z_{i j} .
\end{aligned}
$$

This example demonstrates that for any second order tensor $\mathbf{Z}, \mathcal{R}\{\underline{\mathcal{E}}\{\mathbf{Z}\}\}=\mathbf{Z}$.

\section{Orthogonal Vector States}

This section concerns vector states whose action is to rigidly rotate vectors $\xi \in \mathcal{H}$ about some axis, through some angle. Let $\mathcal{O}^{+}$denote the set of proper orthogonal second order tensors.

Definition 4.1 For any $\mathbf{Q} \in \mathcal{O}^{+}$, let $\mathbf{Q}=\underline{\mathcal{E}}(\mathbf{Q})$. Such a $\mathbf{Q}$ is called a proper orthogonal vector state. The set of all proper othogonal vector states is denoted $\mathcal{Q}$. Note that $\mathcal{Q} \subset \mathcal{V}$. Also, for any $\mathbf{Q} \in \mathcal{O}^{+}$, let $\underline{\mathbf{Q}}^{T}=\underline{\mathcal{E}}\left(\mathbf{Q}^{T}\right)$, the transpose of $\underline{\mathbf{Q}}$. It is immediate that $\underline{\mathbf{Q}} \in \mathcal{Q}$ if and only if $\underline{\mathbf{Q}}^{T} \in \mathcal{Q}$.

The transpose of a proper orthogonal vector state, $\mathbf{Q}^{T}$, inherits the following properties from $\mathbf{Q}^{T}$ :

$$
\begin{array}{ll}
\underline{\mathbf{Q}}^{T}\langle\boldsymbol{\xi}\rangle \cdot \zeta=\xi \cdot \underline{\mathbf{Q}}\langle\zeta\rangle & \forall \boldsymbol{\xi}, \zeta \in \mathcal{H} ; \\
\underline{\mathbf{Q}}^{T} \circ \underline{\mathbf{Q}}=\underline{\mathbf{Q}} \circ \underline{\mathbf{Q}}^{T}=\underline{\mathbf{X}} \quad \forall \underline{\mathbf{Q}} \in \mathcal{Q} .
\end{array}
$$

\section{Derivatives of Functions of States}

The subject of this section is functions of states. Such a function may be scalar-valued or tensor-valued. The quantity that such a function depends on will be written in parentheses, e.g., $\Psi(\underline{\mathbf{B}})$.

Definition 5.1 Let $\Psi$ be a function of a state, $\Psi(\cdot): \mathcal{A}_{m} \rightarrow \mathcal{L}_{n}$. Suppose there exists a state-valued function denoted $\nabla \Psi \in \mathcal{A}_{m+n}$ such that for any $\underline{\mathbf{A}} \in \mathcal{A}_{m}$ and any $\Delta \underline{\mathbf{A}} \in \mathcal{A}_{m}$,

$$
\Psi(\underline{\mathbf{A}}+\Delta \underline{\mathbf{A}})=\Psi(\underline{\mathbf{A}})+\nabla \Psi(\underline{\mathbf{A}}) \bullet \Delta \underline{\mathbf{A}}+o(\|\Delta \underline{\mathbf{A}}\|) .
$$

Then $\Psi$ is said to be differentiable and $\nabla \Psi$ is called the Frechet derivative of $\Psi$. 
The most common situation for present purposes is that $\Psi$ is a scalar-valued function of a vector state. In this case $\nabla \Psi$, if it exists, is a vector state. However, in Section 18 , it will be necessary to consider the Frechet derivative of a second-order tensor-valued function of a vector state; such a derivative is a state of order 3.

Example Let $\Psi(\underline{\mathbf{A}})=3 \underline{\mathbf{A}} \bullet \underline{\mathbf{A}}$ for all $\underline{\mathbf{A}} \in \mathcal{V}$. Then

$$
\begin{aligned}
\Psi(\underline{\mathbf{A}}+\Delta \underline{\mathbf{A}}) & =3(\underline{\mathbf{A}}+\Delta \underline{\mathbf{A}}) \bullet(\underline{\mathbf{A}}+\Delta \underline{\mathbf{A}}) \\
& =3 \underline{\mathbf{A}} \bullet \underline{\mathbf{A}}+6 \underline{\mathbf{A}} \bullet \Delta \underline{\mathbf{A}}+o(\|\Delta \underline{\mathbf{A}}\|),
\end{aligned}
$$

hence $\nabla \Psi(\underline{\mathbf{A}})=6 \underline{\mathbf{A}}$. Thus, $\nabla \underline{\Psi}$ is a vector state.

Notation such as $\partial / \partial \underline{\mathbf{A}}$ will be used to denote a Frechet derivative of a function with respect to some quantity that is contained explicitly within its argument, for example, if we are considering $\Psi(\underline{b \mathbf{A}})$, then $\partial / \partial \underline{\mathbf{A}}$ is defined by

$$
\Psi(\underline{b}(\underline{\mathbf{A}}+\Delta \underline{\mathbf{A}}))=\Psi(\underline{b \mathbf{A}})+\frac{\partial \Psi}{\partial \underline{\mathbf{A}}}(\underline{b \mathbf{A}}) \bullet \Delta \underline{\mathbf{A}}+o(\|\Delta \underline{\mathbf{A}}\|) .
$$

Frechet derivatives have many of the same properties as the more familiar gradients in finite dimensional vector spaces. For future reference, some elementary properties of Frechet derivatives are recorded below, in which $\underline{\mathbf{A}} \in \mathcal{V}, \underline{b} \in \mathcal{S}$, and $\underline{\mathbf{Q}} \in \mathcal{Q}$ :

$$
\begin{gathered}
\frac{\partial \Psi}{\partial \underline{\mathbf{A}}}(\underline{b \mathbf{A}})=\underline{b} \nabla \Psi(\underline{b \mathbf{A}}), \\
\frac{\partial \Psi}{\partial \underline{\mathbf{A}}}(\underline{\mathbf{Q}} \circ \underline{\mathbf{A}})=\underline{\mathbf{Q}}^{T} \circ \nabla \Psi(\underline{\mathbf{Q}} \circ \underline{\mathbf{A}}), \\
\frac{\partial \Psi}{\partial \underline{\mathbf{A}}}(\underline{\mathbf{A}} \circ \underline{\mathbf{Q}})=\nabla \Psi(\underline{\mathbf{A}} \circ \underline{\mathbf{Q}}) \circ \underline{\mathbf{Q}}^{T} .
\end{gathered}
$$

\section{State Fields}

A state field is a state-valued function of position $\mathbf{x}$ (in the reference configuration) and time $t$. The location and time at which particular values of a state field are to be evaluated are shown in square brackets: $\underline{\mathbf{A}}[\mathbf{x}, t]$ and $\underline{B}[\mathbf{x}, t]$.

A position- and time-dependent vector state-valued function $\underline{\Psi}$ of a state $\underline{\mathbf{A}}$ would be written as $\underline{\Psi}[\mathbf{x}, t](\underline{\mathbf{A}})$. Finally, the value of such a field operating on a particular vector $\xi$ would be

$$
\mathbf{v}=\underline{\Psi}[\mathbf{x}, t](\underline{\mathbf{A}})\langle\xi\rangle
$$

but fortunately this full notation is not often necessary.

\section{State Based Peridynamic Theory}

In the state based theory, the equation of motion (1) is replaced by the following:

$$
\rho(\mathbf{x}) \ddot{\mathbf{u}}(\mathbf{x}, t)=\int_{\mathcal{H}_{\mathbf{x}}}\left\{\underline{\mathbf{T}}[\mathbf{x}, t]\left\langle\mathbf{x}^{\prime}-\mathbf{x}\right\rangle-\underline{\mathbf{T}}\left[\mathbf{x}^{\prime}, t\right]\left\langle\mathbf{x}-\mathbf{x}^{\prime}\right\rangle\right\} d V_{\mathbf{x}^{\prime}}+\mathbf{b}(\mathbf{x}, t),
$$


where $\mathcal{H}_{\mathbf{x}}$ is a spherical neighborhood of radius $\delta$ centered at $\mathbf{x}$, and where $\underline{\mathbf{T}}$ is the force vector state field. (How $\underline{\mathbf{T}}$ is determined is the subject of Sections 8-18.) It will be assumed that $\underline{\mathbf{T}}$ is Riemann integrable, and that the integral in (28) converges uniformly. To make the notation more concise, (28) will be abbreviated as

$$
\rho \ddot{\mathbf{u}}=\int_{\mathcal{H}_{\mathbf{x}}}\left\{\underline{\mathbf{T}}\left\langle\mathbf{x}^{\prime}-\mathbf{x}\right\rangle-\underline{\mathbf{T}}^{\prime}\left\langle\mathbf{x}-\mathbf{x}^{\prime}\right\rangle\right\} d V_{\mathbf{x}^{\prime}}+\mathbf{b},
$$

where

$$
\underline{\mathbf{T}}=\underline{\mathbf{T}}[\mathbf{x}, t], \quad \underline{\mathbf{T}}^{\prime}=\underline{\mathbf{T}}\left[\mathbf{x}^{\prime}, t\right] .
$$

To ensure consistency with basic physical principles, it will be required that $\underline{\mathbf{T}}$ satisfy balance of linear momentum for any bounded body $\mathcal{B}$ :

$$
\int_{\mathcal{B}} \rho \ddot{\mathbf{i}}(\mathbf{x}, t) d V_{\mathbf{x}}=\int_{\mathcal{B}} \mathbf{b}(\mathbf{x}, t) d V_{\mathbf{x}} \quad \forall t \geq 0 .
$$

The following proposition asserts that this requirement is automatically satisfied regardless of how $\underline{\mathbf{T}}$ is determined.

Proposition 7.1 Let $\mathcal{B}$ be a bounded body subjected to a body force density field $\mathbf{b}$. Let $\underline{\mathbf{T}}$ be the force vector state field on $\mathcal{B}$, and let (28) hold on $\mathcal{B}$ for all $t \geq 0$. Then (31) holds, i.e., balance of linear momentum is satisfied.

Proof For any $t$, using (29),

$$
\int_{\mathcal{B}}(\rho \ddot{\mathbf{u}}-\mathbf{b}) d V_{\mathbf{x}}=\int_{\mathcal{B}} \int_{\mathcal{H}_{\mathbf{x}}}\left\{\underline{\mathbf{T}}\left\langle\mathbf{x}^{\prime}-\mathbf{x}\right\rangle-\underline{\mathbf{T}}^{\prime}\left\langle\mathbf{x}-\mathbf{x}^{\prime}\right\rangle\right\} d V_{\mathbf{x}^{\prime}} d V_{\mathbf{x}} .
$$

Since $\underline{\mathbf{T}}\left\langle\mathbf{x}^{\prime}-\mathbf{x}\right\rangle=\underline{\mathbf{0}}$ whenever $\mathbf{x}^{\prime} \notin \mathcal{H}_{\mathbf{x}}$, the limits of integration on the inner integral may be changed from $\mathcal{H}_{\mathbf{x}}$ to $\mathcal{B}$. In the second term of the integrand, the dummy variables may be relabeled $\mathbf{x} \leftrightarrow \mathbf{x}^{\prime}$. Also, the order of integration in the second term may be exchanged, leading to

$$
\begin{aligned}
\int_{\mathcal{B}}(\rho \ddot{\mathbf{u}}-\mathbf{b}) d V_{\mathbf{x}} & =\int_{\mathcal{B}} \int_{\mathcal{B}}\left\{\underline{\mathbf{T}}\left\langle\mathbf{x}^{\prime}-\mathbf{x}\right\rangle-\underline{\mathbf{T}}\left\langle\mathbf{x}^{\prime}-\mathbf{x}\right\rangle\right\} d V_{\mathbf{x}^{\prime}} d V_{\mathbf{x}} \\
& =\mathbf{0}
\end{aligned}
$$

so (31) holds.

Similarly, balance of angular momentum in a bounded body $\mathcal{B}$ is required:

$$
\int_{\mathcal{B}} \mathbf{y}(\mathbf{x}, t) \times(\rho \ddot{\mathbf{u}}(\mathbf{x}, t)-\mathbf{b}(\mathbf{x}, t)) d V_{\mathbf{x}}=0 \quad \forall t \geq 0,
$$

where

$$
\mathbf{y}(\mathbf{x}, t)=\mathbf{x}+\mathbf{u}(\mathbf{x}, t) \quad \forall t \geq 0, \mathbf{x} \in \mathcal{B} .
$$

However, unlike the balance of linear momentum, (34) may or may not be satisfied for a particular $\underline{\mathbf{T}}$ field. The first proposition in Section 8 contains a restriction on $\underline{\mathbf{T}}$ that ensures that the balance of angular momentum is satisfied. 


\section{Constitutive Models}

Definition 8.1 Let $\underline{\mathbf{Y}}$ be the deformation vector state field defined by

$$
\underline{\mathbf{Y}}[\mathbf{x}, t]\langle\boldsymbol{\xi}\rangle=\mathbf{y}(\mathbf{x}+\boldsymbol{\xi}, t)-\mathbf{y}(\mathbf{x}, t) \quad \forall \mathbf{x} \in \mathcal{B}, \xi \in \mathcal{H}, t \geq 0
$$

thus $\underline{\mathbf{Y}}\left\langle\mathbf{x}^{\prime}-\mathbf{x}\right\rangle$ is the image of the bond $\mathbf{x}^{\prime}-\mathbf{x}$ under the deformation. Define the reference position scalar state field $\underline{x}$ and the deformation scalar state field $y$ by

$$
\underline{x}=|\underline{\mathbf{X}}|, \quad \underline{y}=|\underline{\mathbf{Y}}| .
$$

(See Fig. 2.)

The following assumption will apply to the remainder of this discussion:

$$
\underline{\mathbf{Y}}\langle\boldsymbol{\xi}\rangle=\mathbf{0} \quad \text { if and only if } \boldsymbol{\xi}=\mathbf{0},
$$

which means that two distinct particles never occupy the same point as the deformation progresses.

A constitutive model in the state based theory is a relation that provides values for the force vector state field in terms of the deformation vector state field and possibly other variables as well. The general form of a constitutive model will be written as

$$
\underline{\mathbf{T}}=\underline{\hat{\mathbf{T}}}(\underline{\mathbf{Y}}, \Lambda)
$$

where $\underline{\hat{\mathbf{T}}}: \mathcal{V} \rightarrow \mathcal{V}$ is bounded and Riemann-integrable on $\mathcal{H}$, and where $\Lambda$ denotes all variables other than the current deformation vector state that $\underline{\mathbf{T}}$ may depend on for some particular material.

Fig. 2 Reference and deformed vector states

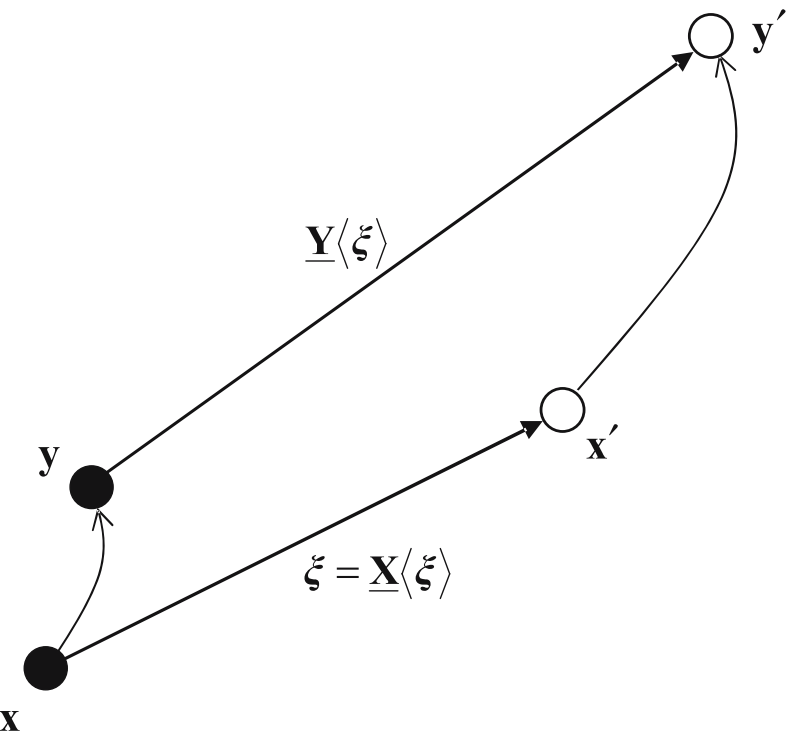


Definition 8.2 If, for a given material, $\underline{\mathbf{T}}$ depends only on $\underline{\mathbf{Y}}$, then the material is called simple, and we write

$$
\underline{\mathbf{T}}=\underline{\hat{\mathbf{T}}}(\underline{\mathbf{Y}})
$$

for some function $\underline{\hat{\mathbf{T}}}: \mathcal{V} \rightarrow \mathcal{V}$.

For present purposes, it will be assumed that all materials are simple. (An exception is discussed in Section 16, which deals with plastic deformation, and therefore inherently involves the history of deformation as well as the current deformation.)

Proposition 8.1 Suppose a constitutive model of the form (39) is such that

$$
\int_{\mathcal{H}} \underline{\mathbf{Y}}\langle\boldsymbol{\xi}\rangle \times \underline{\mathbf{T}}\langle\boldsymbol{\xi}\rangle d V_{\boldsymbol{\xi}}=0 \quad \forall \underline{\mathbf{Y}} \in \mathcal{V} .
$$

Then the balance of angular momentum (34) holds for any deformation of a body $\mathcal{B}$ with this constitutive model.

Proof Suppose (41) holds. Consider any deformation of $\mathcal{B}$. Then, using (29) and (36),

$$
\begin{aligned}
\int_{\mathcal{B}} \mathbf{y} \times(\rho \ddot{\mathbf{u}}-\mathbf{b}) d V_{\mathbf{x}}= & \int_{\mathcal{B}}(\mathbf{x}+\mathbf{u}) \times(\rho \ddot{\mathbf{u}}-\mathbf{b}) d V_{\mathbf{x}} \\
= & \int_{\mathcal{B}} \int_{\mathcal{B}}(\mathbf{x}+\mathbf{u}) \times\left(\underline{\mathbf{T}}\left\langle\mathbf{x}^{\prime}-\mathbf{x}\right\rangle-\underline{\mathbf{T}}^{\prime}\left\langle\mathbf{x}-\mathbf{x}^{\prime}\right\rangle\right) d V_{\mathbf{x}^{\prime}} d V_{\mathbf{x}} \\
= & \int_{\mathcal{B}} \int_{\mathcal{B}}(\mathbf{x}+\mathbf{u}) \times \underline{\mathbf{T}}\left\langle\mathbf{x}^{\prime}-\mathbf{x}\right\rangle d V_{\mathbf{x}^{\prime}} d V_{\mathbf{x}} \\
& -\int_{\mathcal{B}} \int_{\mathcal{B}}(\mathbf{x}+\mathbf{u}) \times \underline{\mathbf{T}^{\prime}}\left\langle\mathbf{x}-\mathbf{x}^{\prime}\right\rangle d V_{\mathbf{x}^{\prime}} d V_{\mathbf{x}} \\
= & \int_{\mathcal{B}} \int_{\mathcal{B}}(\mathbf{x}+\mathbf{u}) \times \underline{\mathbf{T}}\left\langle\mathbf{x}^{\prime}-\mathbf{x}\right\rangle d V_{\mathbf{x}^{\prime}} d V_{\mathbf{x}} \\
& -\int_{\mathcal{B}} \int_{\mathcal{B}}\left(\mathbf{x}^{\prime}+\mathbf{u}^{\prime}\right) \times \underline{\mathbf{T}}\left\langle\mathbf{x}^{\prime}-\mathbf{x}\right\rangle d V_{\mathbf{x}} d V_{\mathbf{x}^{\prime}} \\
= & -\int_{\mathcal{B}} \int_{\mathcal{B}}\left(\left(\mathbf{x}^{\prime}+\mathbf{u}^{\prime}\right)-(\mathbf{x}+\mathbf{u})\right) \times \underline{\mathbf{T}}\left\langle\mathbf{x}^{\prime}-\mathbf{x}\right\rangle d V_{\mathbf{x}^{\prime}} d V_{\mathbf{x}} \\
= & -\int_{\mathcal{B}} \int_{\mathcal{B}}\left(\mathbf{y}^{\prime}-\mathbf{y}\right) \times \underline{\mathbf{T}}\left\langle\mathbf{x}^{\prime}-\mathbf{x}\right\rangle d V_{\mathbf{x}^{\prime}} d V_{\mathbf{x}} \\
= & -\int_{\mathcal{B}} \int_{\mathcal{H}} \underline{\mathbf{Y}}\langle\xi\rangle \times \underline{\mathbf{T}}\langle\xi\rangle d V_{\xi} d V_{\mathbf{x}}=\mathbf{0},
\end{aligned}
$$

so (34) holds. A change of variables $\mathbf{x} \leftrightarrow \mathbf{x}^{\prime}$ is used in the fourth step above. The change in order of integration that appears in the fifth step is permitted under the previously stated assumption that such integrals converge uniformly.

The proposition just proved means that if the force state for each individual $\mathbf{x}$ in the body contains no net moment, then the body as a whole obeys the balance of angular momentum globally. This represents progress, because it reduces (34), which would 
be unwieldy if we had to prove that it holds for every possible deformation of a body, to a much more manageable condition on the constitutive model.

Definition 8.3 Let the deformed direction vector state $\underline{\mathbf{M}}$ be the unit state valued function defined by

$$
\underline{\mathbf{M}}(\underline{\mathbf{Y}})=\operatorname{Dir} \underline{\mathbf{Y}} \quad \forall \underline{\mathbf{Y}} \in \mathcal{V},
$$

thus $\underline{\mathbf{M}}(\underline{\mathbf{Y}})\left\langle\mathbf{x}^{\prime}-\mathbf{x}\right\rangle$ is a unit vector that points from the deformed position of $\mathbf{x}$ toward the deformed position of $\mathbf{x}^{\prime}$.

Definition 8.4 If a material has the property that for any deformation there exists a scalar state $\underline{t}$ such that

$$
\underline{\mathbf{T}}=\underline{t} \underline{\mathbf{M}}
$$

then the material is called ordinary, and $\underline{t}$ is called the scalar force state field. Otherwise the material is called non-ordinary. (Schematics of ordinary and nonordinary material models are shown in Fig. 3.)

In an ordinary material, (44) implies

$$
(\operatorname{Dir} \underline{\mathbf{T}})\langle\boldsymbol{\xi}\rangle=(\operatorname{Dir} \underline{\mathbf{Y}})\langle\boldsymbol{\xi}\rangle \quad \text { whenever } \quad \underline{t}\langle\boldsymbol{\xi}\rangle \neq 0 .
$$

Proposition 8.2 Let $\mathcal{B}$ be a body composed of an ordinary material and subjected to a body force density field $\mathbf{b}$. Let $\underline{\mathbf{T}}$ be the force vector state field in $\mathcal{B}$. Then (34) holds, i.e., balance of angular momentum is satisfied.

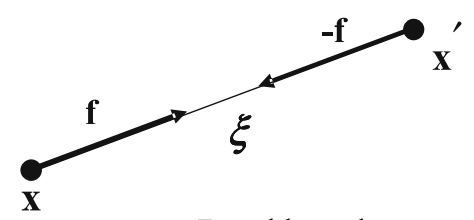

Bond based

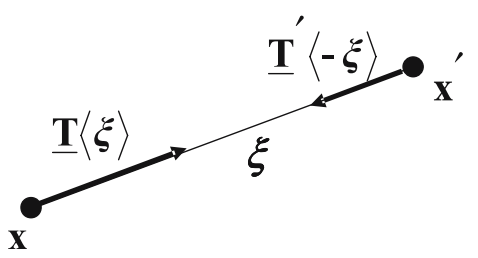

Ordinary state based

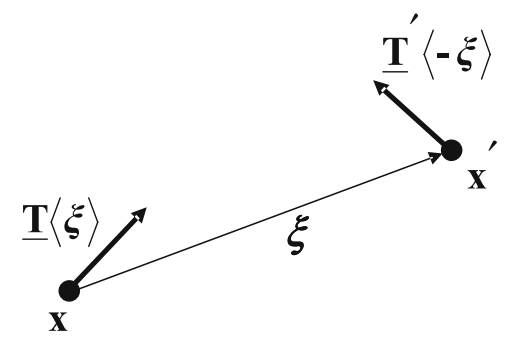

Non-ordinary state based

Fig. 3 Schematics of bond based, ordinary, and non-ordinary material response. All three of these satisfy balance of linear momentum, and the first two always satisfy balance of angular momentum 
Proof Using (14) and (43), taking the vector cross product of $\underline{\mathbf{Y}}$ with $\underline{\mathbf{T}}$ leads to

$$
\begin{aligned}
\underline{\mathbf{Y}}\langle\boldsymbol{\xi}\rangle \times \underline{\mathbf{T}}\langle\boldsymbol{\xi}\rangle & =\underline{\mathbf{Y}}\langle\boldsymbol{\xi}\rangle \times \underline{t}\langle\boldsymbol{\xi}\rangle \underline{\mathbf{M}}\langle\boldsymbol{\xi}\rangle \\
& =\underline{\mathbf{Y}}\langle\boldsymbol{\xi}\rangle \times \underline{t}\langle\boldsymbol{\xi}\rangle\left(\frac{\underline{\mathbf{Y}}\langle\boldsymbol{\xi}\rangle}{|\underline{\mathbf{Y}}|\langle\boldsymbol{\xi}\rangle}\right)=\mathbf{0}
\end{aligned}
$$

provided $\boldsymbol{\xi} \neq \mathbf{0}$. It is also true that $\underline{\mathbf{Y}}\langle\mathbf{0}\rangle \times \underline{\mathbf{T}}\langle\mathbf{0}\rangle$ because of the assumption (38). So, the requirement of Proposition 8.1 is satisfied, and therefore (34) holds.

The fact that ordinary materials satisfy balance angular momentum is also evident geometrically because in such materials, pairs of particles apply force vectors to each other that are opposite in direction, equal in magnitude, and parallel to their relative deformed position. Note that the above proposition assumes nothing about what $\underline{t}$ depends on; only the direction is important.

Example Consider a material for which there exists a scalar-valued function $f(\cdot, \cdot)$ : $\mathbb{R}^{3} \times \mathbb{R}^{3} \rightarrow \mathbb{R}$ such that $f(-\boldsymbol{\eta},-\boldsymbol{\xi})=f(\boldsymbol{\eta}, \boldsymbol{\xi})$ for all $\boldsymbol{\eta}$ and all $\boldsymbol{\xi}$. Then define an ordinary material by

$$
\underline{t}[\mathbf{x}, t]\left\langle\mathbf{x}^{\prime}-\mathbf{x}\right\rangle=\frac{1}{2} f\left(\mathbf{u}^{\prime}-\mathbf{u}, \mathbf{x}^{\prime}-\mathbf{x}\right) \quad \forall \mathbf{x} \in \mathcal{B}, \mathbf{x}^{\prime} \in \mathcal{H}_{\mathbf{x}}, t \geq 0 .
$$

In this case, the equation of motion (28) is equivalent to (1), demonstrating that the bond-based theory is a special case of the state-based theory.

As noted above, ordinariness is a sufficient condition for the balance of linear and angular momenta to be satisfied. In a non-ordinary material, balance of angular momentum is not automatically satisfied, and this provides a restriction on the form of $\underline{\mathbf{T}}$. Yet this requirement can sometimes be met with appropriate constitutive models, and non-ordinary materials can be reasonable and interesting. An example of a non-ordinary material is given in Section 17.

If a material is ordinary, we can write

$$
\underline{t}=\hat{t}(\underline{\mathbf{Y}}) .
$$

for some function $\underline{\hat{t}}: \mathcal{V} \rightarrow \mathcal{S}$.

Definition 8.5 If an ordinary material has the property that there exists a scalar statevalued function $\underline{\tau}: \mathcal{S} \rightarrow \mathcal{S}$ such that

$$
\underline{\hat{t}}(\underline{\mathbf{Y}})=\underline{\tau}(|\underline{\mathbf{Y}}|) \quad \forall \underline{\mathbf{Y}} \in \mathcal{V},
$$

then the material is called mobile and $\underline{\tau}$ is called the mobile force function.

Mobility of a material does not imply that the bonds act independently of each other, i.e., that the material is bond-based. It means that the magnitudes of the forces in $\underline{\mathbf{T}}$ depend on the deformed bond lengths but not on rotation of the bonds. In a mobile material, it is entirely possible that a change in length in bond $\xi$ affects the force in another bond $\zeta$.

By definition, mobility assumes ordinariness. But in general, ordinariness does not imply mobility. (Ordinariness is a condition on the direction of the forces, while 
mobility is a condition on what these forces can depend on.) However, it will be shown in Section 11 that ordinariness in an elastic material (to be defined later) does imply mobility.

\section{Isotropy and Objectivity}

Material isotropy means that there is no special direction in the reference configuration with respect to material response. In an isotropic material, rotation of the body prior to applying a deformation leaves the resulting force vector state unchanged, motivating the following definition.

Definition 9.1 A material is isotropic if

$$
\underline{\hat{\mathbf{T}}}(\underline{\mathbf{Y}} \circ \underline{\mathbf{Q}})=\underline{\hat{\mathbf{T}}}(\underline{\mathbf{Y}}) \circ \underline{\mathbf{Q}} \quad \forall \underline{\mathbf{Y}} \in \mathcal{V}, \forall \underline{\mathbf{Q}} \in \mathcal{Q} .
$$

Before going further, it will be helpful to establish the following intermediate results.

Lemma 9.1 For any $\underline{\mathbf{Y}} \in \mathcal{V}$ and $\underline{\mathbf{Q}} \in \mathcal{Q}$,

(1)

$$
\underline{\mathbf{M}}(\underline{\mathbf{Y}} \circ \underline{\mathbf{Q}})=\underline{\mathbf{M}}(\underline{\mathbf{Y}}) \circ \underline{\mathbf{Q}}
$$

and (2)

$$
\underline{\mathbf{M}}(\underline{\mathbf{Q}} \circ \underline{\mathbf{Y}})=\underline{\mathbf{Q}} \circ \underline{\mathbf{M}}(\underline{\mathbf{Y}}) .
$$

Proof By (6), (14), and (43),

$$
\underline{\mathbf{M}}(\underline{\mathbf{Y}} \circ \underline{\mathbf{Q}})\langle\xi\rangle=\frac{(\underline{\mathbf{Y}} \circ \underline{\mathbf{Q}})\langle\xi\rangle}{|(\underline{\mathbf{Y}} \circ \underline{\mathbf{Q}})\langle\xi\rangle|}=\frac{\underline{\mathbf{Y}}\langle\underline{\mathbf{Q}}\langle\boldsymbol{\xi}\rangle\rangle}{|\underline{\mathbf{Y}}\langle\underline{\mathbf{Q}} \xi\rangle\rangle \mid}=(\underline{\mathbf{M}}(\underline{\mathbf{Y}}) \circ \underline{\mathbf{Q}})\langle\xi\rangle,
$$

proving (1). (2) follows similarly.

Proposition 9.1 An ordinary material is isotropic if and only if

$$
\underline{\hat{t}}(\underline{\mathbf{Y}} \circ \underline{\mathbf{Q}})=\underline{\hat{t}}(\underline{\mathbf{Y}}) \circ \underline{\mathbf{Q}} \quad \forall \underline{\mathbf{Y}} \in \mathcal{V}, \forall \underline{\mathbf{Q}} \in \mathcal{Q} .
$$

Proof Suppose a given ordinary material is isotropic. Then for any $\underline{\mathbf{Y}} \in \mathcal{V}$, any $\underline{\mathbf{Q}} \in$ $\mathcal{Q}$, and any $\xi \in \mathcal{H},(6),(44)$ and (50) imply

$$
\underline{\mathbf{M}}(\underline{\mathbf{Y}} \circ \underline{\mathbf{Q}}) \hat{t}(\underline{\mathbf{Y}} \circ \underline{\mathbf{Q}})=(\underline{\mathbf{M}}(\underline{\mathbf{Y}}) \underline{\hat{t}}(\underline{\mathbf{Y}})) \circ \underline{\mathbf{Q}}=(\underline{\mathbf{M}}(\underline{\mathbf{Y}}) \circ \underline{\mathbf{Q}})(\underline{\hat{t}}(\underline{\mathbf{Y}}) \circ \underline{\mathbf{Q}}) .
$$

But by Lemma 9.1, the right hand side of this may be rewritten as

$$
(\underline{\mathbf{M}}(\underline{\mathbf{Y}}) \circ \underline{\mathbf{Q}})(\underline{\hat{t}}(\underline{\mathbf{Y}}) \circ \underline{\mathbf{Q}})=\underline{\mathbf{M}}(\underline{\mathbf{Y}} \circ \underline{\mathbf{Q}})(\underline{\hat{t}}(\underline{\mathbf{Y}}) \circ \underline{\mathbf{Q}}) .
$$

Comparing the left hand side of (55) with the right hand side of (56) establishes (54). Reversing the steps proves the converse.

To motivate the requirement for a constitutive model to be objective, consider two experiments: (1) deform a body, rotate it, then evaluate the force state; and (2) deform the body, evaluate the force state, then rotate force state. The requirement 
for objectivity is that the force vector state from resulting from these two experiments be equal, so objectivity is defined as follows:

Definition 9.2 A material is objective if

$$
\underline{\hat{\mathbf{T}}}(\underline{\mathbf{Q}} \circ \underline{\mathbf{Y}})=\underline{\mathbf{Q}} \circ \underline{\hat{\mathbf{T}}}(\underline{\mathbf{Y}}) \quad \forall \underline{\mathbf{Y}} \in \mathcal{V}, \forall \underline{\mathbf{Q}} \in \mathcal{Q} .
$$

Proposition 9.2 An ordinary material is objective if and only if

$$
\underline{\hat{t}}(\underline{\mathbf{Q}} \circ \underline{\mathbf{Y}})=\underline{\hat{t}}(\underline{\mathbf{Y}}) \quad \forall \underline{\mathbf{Y}} \in \mathcal{V}, \forall \underline{\mathbf{Q}} \in \mathcal{Q} .
$$

Proof Suppose a given ordinary material is objective. From (2) of Lemma 9, (57) and (44), it follows that for any $\underline{\mathbf{Y}} \in \mathcal{V}$ and $\underline{\mathbf{Q}} \in \mathcal{Q}$,

$$
\underline{\mathbf{M}}(\underline{\mathbf{Q}} \circ \underline{\mathbf{Y}}) \underline{\hat{t}}(\underline{\mathbf{Q}} \circ \underline{\mathbf{Y}})=\underline{\mathbf{Q}} \circ(\underline{\mathbf{M}}(\underline{\mathbf{Y}}) \underline{\hat{t}}(\underline{\mathbf{Y}}))=\underline{\mathbf{M}}(\underline{\mathbf{Q}} \circ \underline{\mathbf{Y}}) \underline{\hat{t}}(\underline{\mathbf{Y}})
$$

which implies (58). Reversing the steps proves the converse.

Proposition 9.3 If a material is ordinary and mobile, then it is objective.

Proof Suppose a material is ordinary and mobile. Then by (49) and the fact that $\underline{\mathbf{Q}}$ leaves vector lengths unchanged,

$$
\hat{t}(\underline{\mathbf{Y}})=\underline{\tau}(|\underline{\mathbf{Y}}|)=\underline{\tau}(|\underline{\mathbf{Q}} \circ \underline{\mathbf{Y}}|)=\hat{t}(\underline{\mathbf{Q}} \circ \underline{\mathbf{Y}}) \quad \forall \underline{\mathbf{Y}} \in \mathcal{V}, \underline{\mathbf{Q}} \in \mathcal{Q} .
$$

so $(58)$ is satisfied.

The converse of this statement is not true, that is, objectivity in an ordinary material does not imply mobility. A counterexample to this converse is a material in which $\hat{t}(\underline{\mathbf{Y}})\langle\boldsymbol{\xi}\rangle=\underline{\mathbf{Y}}\langle\boldsymbol{\xi}\rangle \cdot \underline{\mathbf{Y}}\langle-\boldsymbol{\xi}\rangle$, which is objective but not mobile (because the force depends on angle changes rather than just length changes).

\section{Elastic Materials}

Definition 10.1 A material is elastic if there exists a differentiable scalar valued function $W(\cdot): \mathcal{V} \rightarrow \mathbb{R}$ such that

$$
\underline{\mathbf{T}}=\underline{\hat{\mathbf{T}}}(\underline{\mathbf{Y}})=\nabla W(\underline{\mathbf{Y}}) \quad \forall \underline{\mathbf{Y}} \in \mathcal{V} .
$$

If it exists, $W$ is called the strain energy density function for the material. (See (23) for the meaning of the symbol $\nabla$.)

It will now be demonstrated that elastic materials in the present theory have properties similar to those of elastic materials in the classical theory in regard to energy balance. For a body $\mathcal{B}$ composed of an elastic material subjected to a body force density field $\mathbf{b}$, at any time $t$, define

$$
K(t)=\frac{1}{2} \int_{\mathcal{B}} \rho \dot{\mathbf{u}} \cdot \dot{\mathbf{u}} d V, \quad U(t)=\int_{0}^{t} \int_{\mathcal{B}} \mathbf{b} \cdot \dot{\mathbf{u}} d V d t^{\prime}, \quad \Phi(t)=\int_{\mathcal{B}} W d V,
$$

representing total kinetic energy, total external work, and total strain energy respectively. 
Proposition 10.1 Suppose a bounded body composed of an elastic material is subjected to a body force density field $\mathbf{b}$, and $K(0)=U(0)=\Phi(0)=0$. Then

$$
U(t)=\Phi(t)+K(t) \quad \forall t \geq 0 .
$$

Proof In a body (not necessarily elastic) subjected to a body force density field $\mathbf{b}$, take the vector dot product of both sides of (29) with $\dot{\mathbf{u}}$ and integrate over the body to obtain

$$
\int_{\mathcal{B}}(\rho \ddot{\mathbf{u}}-\mathbf{b}) \cdot \dot{\mathbf{u}} d V_{\mathbf{x}}=\int_{\mathcal{B}} \int_{\mathcal{H}_{\mathbf{x}}}\left\{\underline{\mathbf{T}}\left\langle\mathbf{x}^{\prime}-\mathbf{x}\right\rangle-\underline{\mathbf{T}}^{\prime}\left\langle\mathbf{x}-\mathbf{x}^{\prime}\right\rangle\right\} \cdot \dot{\mathbf{u}} d V_{\mathbf{x}^{\prime}} d V_{\mathbf{x}}
$$

where the following abbreviations are used:

$$
\underline{\mathbf{T}}=\underline{\mathbf{T}}[\mathbf{x}, t], \quad \underline{\mathbf{T}}^{\prime}=\underline{\mathbf{T}}\left[\mathbf{x}^{\prime}, t\right], \quad \mathbf{u}=\mathbf{u}(\mathbf{x}, t), \quad \mathbf{u}^{\prime}=\mathbf{u}\left(\mathbf{x}^{\prime}, t\right) .
$$

Noting that $\underline{\mathbf{T}}\left\langle\mathbf{x}^{\prime}-\mathbf{x}\right\rangle=\mathbf{0}$ whenever $\mathbf{x}^{\prime} \notin \mathcal{H}_{\mathbf{x}}$, it follows from (64) that

$$
\begin{aligned}
\int_{\mathcal{B}}(\rho \ddot{\mathbf{u}}-\mathbf{b}) \cdot \dot{\mathbf{u}} d V_{\mathbf{x}} & =\int_{\mathcal{B}} \int_{\mathcal{B}} \underline{\mathbf{T}}\left\langle\mathbf{x}^{\prime}-\mathbf{x}\right\rangle \cdot \dot{\mathbf{u}} d V_{\mathbf{x}} d V_{\mathbf{x}^{\prime}}-\int_{\mathcal{B}} \int_{\mathcal{B}} \underline{\mathbf{T}^{\prime}}\left\langle\mathbf{x}-\mathbf{x}^{\prime}\right\rangle \cdot \dot{\mathbf{u}} d V_{\mathbf{x}} d V_{\mathbf{x}^{\prime}} \\
& =\int_{\mathcal{B}} \int_{\mathcal{B}} \underline{\mathbf{T}}\left\langle\mathbf{x}^{\prime}-\mathbf{x}\right\rangle \cdot \dot{\mathbf{u}} d V_{\mathbf{x}} d V_{\mathbf{x}^{\prime}}-\int_{\mathcal{B}} \int_{\mathcal{B}} \underline{\mathbf{T}}\left\langle\mathbf{x}^{\prime}-\mathbf{x}\right\rangle \cdot \dot{\mathbf{u}}^{\prime} d V_{\mathbf{x}} d V_{\mathbf{x}^{\prime}} \\
& =-\int_{\mathcal{B}} \int_{\mathcal{B}} \underline{\mathbf{T}}\left\langle\mathbf{x}^{\prime}-\mathbf{x}\right\rangle \cdot\left(\dot{\mathbf{u}}^{\prime}-\dot{\mathbf{u}}\right) d V_{\mathbf{x}^{\prime}} d V_{\mathbf{x}} \\
& =-\int_{\mathcal{B}} \int_{\mathcal{B}} \underline{\mathbf{T}}\langle\xi\rangle \cdot \underline{\dot{\mathbf{Y}}}\langle\xi\rangle d V_{\xi} d V_{\mathbf{x}} \\
& =-\int_{\mathcal{B}} \underline{\mathbf{T}} \bullet \underline{\dot{\mathbf{Y}}} d V_{\mathbf{x}} .
\end{aligned}
$$

where (36) has been used in the second to last step. The result (66) holds for any constitutive model. To specialize it to an elastic material, combine it with (61) to obtain

$$
\int_{\mathcal{B}}(\rho \ddot{\mathbf{u}}-\mathbf{b}) \cdot \dot{\mathbf{u}} d V_{\mathbf{x}}=-\int_{\mathcal{B}} \nabla W \bullet \underline{\dot{\mathbf{Y}}} d V_{\mathbf{x}}=-\int_{\mathcal{B}} \dot{W} d V_{\mathbf{x}} .
$$

Since $\partial(\dot{\mathbf{u}} \cdot \dot{\mathbf{u}}) / \partial t=2 \ddot{\mathbf{i}} \cdot \dot{\mathbf{u}}$, the conclusion from (67) is that

$$
\dot{U}=\dot{\Phi}+\dot{K} \text {. }
$$

Integrating the three quantities in (68) over time proves the result (63).

Proposition 10.1 is the familiar statement that work done on an elastic body through external forces is partitioned into kinetic energy and strain energy. It further shows that in an elastic body, the strain energy density defined in (61) represents the appropriate physics concept of stored energy density, as opposed to being merely an abstract mathematical entity. Finally, it also shows that in a quasi-static deformation, i.e., $K \equiv 0$, then the external work is recoverable by reversing the deformation.

Using the properties of Frechet derivatives in (26), along with (50), (57), and (61), the following results are immediate: 
Proposition 10.2 An elastic material is isotropic if and only if

$$
W(\underline{\mathbf{Y}} \circ \underline{\mathbf{Q}})=W(\underline{\mathbf{Y}}) \quad \forall \underline{\mathbf{Y}} \in \mathcal{V}, \forall \underline{\mathbf{Q}} \in \mathcal{Q},
$$

Proposition 10.3 An elastic material is objective if and only if

$$
W(\underline{\mathbf{Q}} \circ \underline{\mathbf{Y}})=W(\underline{\mathbf{Y}}) \quad \forall \underline{\mathbf{Y}} \in \mathcal{V}, \forall \underline{\mathbf{Q}} \in \mathcal{Q} .
$$

\section{Ordinary Elastic Materials}

Proposition 11.1 If a material is ordinary and elastic, then:

(1) It is mobile.

(2) There exists a scalar-valued function $w: \mathcal{S} \rightarrow \mathbb{R}$ such that

$$
W(\underline{\mathbf{Y}})=w(\underline{y}) \quad \underline{y}=|\underline{\mathbf{Y}}|, \quad \forall \underline{\mathbf{Y}} \in \mathcal{V} .
$$

(3) For this w,

$$
\underline{\tau}(\underline{y})=\nabla w(\underline{y}) \quad \forall \underline{\mathbf{Y}} \in \mathcal{V} .
$$

Proof Suppose a given material is ordinary and elastic. Then from (44) and (61),

$$
\nabla W(\underline{\mathbf{Y}})=\underline{\mathbf{M} t}, \quad \underline{\mathbf{M}}=\underline{\mathbf{M}}(\underline{\mathbf{Y}}), \quad \underline{t}=\underline{\hat{t}}(\underline{\mathbf{Y}}),
$$

where

$$
\underline{\mathbf{Y}}=\underline{\mathbf{M}} \underline{y} .
$$

Consider an increment in the deformation state $\Delta \underline{\mathbf{Y}}$, and let

$$
\underline{\mathbf{Y}}+\Delta \underline{\mathbf{Y}}=(\underline{\mathbf{M}}+\Delta \underline{\mathbf{M}})(\underline{y}+\Delta \underline{y}) .
$$

From the definition of the Frechet derivative (23), (73), and (74), and dropping second order terms,

$$
\begin{aligned}
\Delta W & =\nabla W \bullet \Delta(\underline{\mathbf{M}} \underline{y}) \\
& =(\underline{\mathbf{M} t}) \bullet(\underline{y} \Delta \underline{\mathbf{M}}+\underline{\mathbf{M}} \Delta \underline{y}) \\
& =(\underline{\mathbf{M} t}) \bullet(\underline{y} \Delta \underline{\mathbf{M}})+(\underline{\mathbf{M} t}) \bullet(\underline{\mathbf{M}} \Delta \underline{y}) \\
& =\underline{t} \bullet \Delta \underline{y} .
\end{aligned}
$$

The simplification in the last step results from the properties of unit states,

$$
\mathbf{M}\langle\boldsymbol{\xi}\rangle \cdot \Delta \mathbf{M}\langle\boldsymbol{\xi}\rangle=0, \quad \mathbf{M}\langle\boldsymbol{\xi}\rangle \cdot \mathbf{M}\langle\boldsymbol{\xi}\rangle=1, \quad \forall \boldsymbol{\xi} \in \mathcal{H} .
$$

Moreover, if $W$ is regarded as a function of the two states $\underline{\mathbf{M}}$ and $\underline{y}$ separately, it follows that

$$
\Delta W=\frac{\partial W}{\partial \underline{\mathbf{M}}} \bullet \Delta \underline{\mathbf{M}}+\frac{\partial W}{\partial \underline{y}} \bullet \Delta \underline{y} .
$$


Since the last expression in (76) must equal the right hand side of (78) for all choices of $\Delta \underline{\mathbf{M}}$ and $\Delta \underline{y}$, the conclusion is that

$$
\frac{\partial W}{\partial \underline{\mathbf{M}}}=\underline{\mathbf{0}}, \quad \underline{t}=\frac{\partial W}{\partial \underline{y}}
$$

from which (1), (2), and (3) all follow immediately.

It was shown near the end of Section 9 that mobility (with or without elasticity) implies objectivity. This, together with Proposition 11.1, proves the following:

\section{Corollary 11.1 If a material is ordinary and elastic, then it is objective.}

It is not true that mobility implies isotropy, with or without elasticity. A counterexample is any mobile material in which $\underline{\tau}\langle\boldsymbol{\xi}\rangle$ depends explicitly on the orientation of $\boldsymbol{\xi}$ with respect to some coordinate frame.

\section{Correspondence}

In practice, one may encounter a situation in which an elastic material model from the classical theory is given, and it is desired to specify a state based peridynamic constitutive model that results in the same physical properties in those situations in which a meaningful comparison between the two can be made. To make this more precise, the following definition will be introduced:

Definition 12.1 Let $\mathcal{L}^{+}$be the set of all second order tensors with positive determinant. Let $\mathbf{F} \in \mathcal{L}^{+}$denote the deformation gradient tensor. Consider a function $\Omega(\cdot): \mathcal{L}^{+} \rightarrow \mathbb{R}$ that maps any $\mathbf{F}$ to the corresponding strain energy density in the classical (local) theory of elasticity. Suppose there is a peridynamic strain energy density function $W(\cdot): \mathcal{V} \rightarrow \mathbb{R}$ such that for some choice of $\mathbf{F} \in \mathcal{L}^{+}$,

$$
\underline{\mathbf{Y}}\langle\boldsymbol{\xi}\rangle=\mathbf{F} \boldsymbol{\xi} \quad \forall \boldsymbol{\xi} \in \mathcal{H} \quad \Longrightarrow \quad W(\underline{\mathbf{Y}})=\Omega(\mathbf{F}) .
$$

Then the peridynamic constitutive model corresponds to the classical constitutive model at $\mathbf{F}$.

If a peridynamic material corresponds to a classical material, this means the two are "the same" for purposes of homogeneous deformations of a homogeneous body with deformation gradient F. Yet correspondence says or assumes nothing about what happens in all other deformations and all other bodies. However, in Section 18, a much stronger connection will be made between a peridynamic and a classical model, in which an approximate value of $\mathbf{F}$ is evaluated for any $\underline{\mathbf{Y}}$ (using the idea of reduction to a tensor). Then this approximate $\mathbf{F}$ is used directly in the classical model $\Omega$ to evaluate the force state $\underline{\mathbf{T}}$.

The primary use of the idea of correspondence is in the calibration of peridynamic material models, i.e., the evaluation of parameters in a model so that it gives the same results as the classical model for a given homogeneous deformation. Examples of this calibration process appear in the following sections. 


\section{Decomposition of States}

In this section, a means of separating a deformation state into parts representing the net volume change, and the remainder after separating out the volume change, will be described. This paves the way for independently representing material dependence on volume changes and shears, a concept that is well established in the classical theory. It also permits a notion of plastic incompressibility to be introduced.

In this section, the material is assumed to be ordinary and mobile. $\underline{\omega}$ is an influence function (see Definition 3.2).

Definition 13.1 Define the extension scalar state $\underline{e} \in \mathcal{S}$ by

$$
\underline{e}=\underline{y}-\underline{x}, \quad \underline{y}=|\underline{\mathbf{Y}}|, \quad \underline{x}=|\underline{\mathbf{X}}| .
$$

Note that in general, $\underline{e} \neq|\underline{\mathbf{Y}}-\underline{\mathbf{X}}|$. The value of any $\underline{e}\langle\boldsymbol{\xi}\rangle$ is the change in length of the bond $\xi$ due to deformation.

Definition 13.2 The weighted volume $m$ is the scalar defined by

$$
m=(\underline{\omega x}) \bullet \underline{x} .
$$

Definition 13.3 Define a scalar-valued function called the dilatation $\hat{\theta}: \mathcal{S} \rightarrow \mathbb{R}$ by

$$
\hat{\theta}(\underline{e})=\frac{3}{m}(\underline{\omega x}) \bullet \underline{e} \quad \forall \underline{e} \in \mathcal{S} .
$$

For a small deformation, $\theta=\hat{\theta}(\underline{e})$ is a measure of the volumetric strain. To see this, suppose there is an isotropic deformation of the form $\underline{\mathbf{Y}}=\left(1+\varepsilon_{0}\right) \underline{\mathbf{X}}$ for all $\mathbf{x}$, where $\varepsilon_{0}$ is a constant scalar, $\left|\varepsilon_{0}\right|<<1$. Then (83) implies $\theta=3 \varepsilon_{0}$, which is the same as would be obtained by evaluating the trace of the linearized strain tensor $\epsilon_{i j}$ in the classical theory. It is well known that this $\epsilon_{k k}$ geometrically represents the volumetric strain for sufficiently small displacements.

For future reference, note that from (83) and the definition of Frechet derivative (23),

$$
\nabla \hat{\theta}(\underline{e})=\frac{3 \underline{\omega x}}{m}
$$

Definition 13.4 Define the isotropic part and the deviatoric part of $\underline{e}$ respectively by

$$
\underline{e}^{\mathrm{i}}=\frac{\theta \underline{x}}{3}, \quad \underline{e}^{\mathrm{d}}=\underline{e}-\underline{e}^{\mathrm{i}}, \quad \theta=\hat{\theta}(\underline{e}) . \quad \forall \underline{t} \in \mathcal{S} .
$$

Definition 13.5 Let the force scalar state be given by $\underline{t}=\underline{t} \underline{\hat{t}}(\underline{)})$. Define the peridynamic pressure by

$$
p=-\frac{\underline{t} \bullet \underline{x}}{3}
$$

Definition 13.6 Define the co-isotropic part and the co-deviatoric part of $\underline{t}$ respectively by

$$
\underline{t}^{\mathrm{i}}=\frac{-3 p}{m} \underline{\omega x}, \quad \underline{t}^{\mathrm{d}}=\underline{t}-\underline{t}^{\mathrm{i}}, \quad \forall \underline{t} \in \mathcal{S} .
$$


Note that the isotropic part of $\underline{e}$ and the co-isotropic part of $\underline{t}$ contain an important difference: the latter includes $\underline{\omega}$, while the former does not. This is intentional and helpful, because it allows an influence function to be used to select what bonds within a deformation state are to participate in determining the force state. For example, suppose a void is present somewhere within the horizon of a point $\mathbf{x}$ (which is not contained in the void). If $\underline{\omega}$ is chosen so that it vanishes for all bonds $\xi$ that connect $\mathbf{x}$ to $\mathbf{x}^{\prime}$ located within the void, then the first of (87) shows that $\underline{t}^{i}$ also vanishes for these $\xi$. Similarly, if multiple materials are present within the horizon, $\underline{\omega}$ can be chosen to select one material or the other.

Now consider an incremental change to the deformation state $\Delta \underline{\mathbf{Y}}$ that results in the following increments:

$$
\begin{gathered}
\Delta \underline{e}=|\underline{\mathbf{Y}}+\Delta \underline{\mathbf{Y}}|-|\underline{\mathbf{Y}}|, \quad \Delta \theta=\frac{3}{m}(\underline{\omega x}) \bullet \Delta \underline{e}, \\
\Delta \underline{e}^{\mathrm{i}}=\frac{\Delta \theta \underline{x}}{3}, \quad \Delta \underline{e}^{\mathrm{d}}=\Delta \underline{e}-\Delta \underline{e}^{\mathrm{i}} .
\end{gathered}
$$

The following lemma records some intermediate results that follow immediately from (82-89).

Lemma 13.1 Let a deformation state $\underline{\mathbf{Y}}$ and an increment $\Delta \underline{\mathbf{Y}}$ be given. Then:

$$
\nabla \hat{\theta} \bullet \Delta \underline{e}^{\mathrm{d}}=0, \quad \underline{t}^{\mathrm{i}} \bullet \Delta \underline{e}^{\mathrm{d}}=0, \quad \underline{t}^{\mathrm{d}} \bullet \Delta \underline{e}^{\mathrm{i}}=0 .
$$

Now assume that the material is elastic as well as ordinary and mobile. Making a slight modification to the notation, let the strain energy density be given by $W(\underline{e})$. Thus, from Proposition 11.1,

$$
\underline{t}=\underline{\hat{t}}(\underline{e})=\nabla W(\underline{e}) .
$$

From (23), (87), (89), and (91),

$$
\Delta W=\left(\underline{t}^{\mathrm{i}}+\underline{t}^{\mathrm{d}}\right) \bullet\left(\Delta \underline{e}^{\mathrm{i}}+\Delta \underline{e}^{\mathrm{d}}\right) .
$$

Expanding this and applying the second and third of (90) leads to

$$
\Delta W=\underline{t}^{\mathrm{i}} \bullet \Delta \underline{e}^{\mathrm{i}}+\underline{t}^{\mathrm{d}} \bullet \Delta \underline{e}^{\mathrm{d}} .
$$

By virtue of the decomposition in (85), W may be regarded as a function of $\left\{\underline{e}^{\mathrm{i}}, \underline{e}^{\mathrm{d}}\right\}$ or, since $\underline{e}^{\mathrm{i}}$ depends on the deformation only through $\theta$, as a function of $\left\{\theta, \underline{e}^{\mathrm{d}}\right\}$. Thus,

$$
\Delta W=\frac{\partial W}{\partial \underline{e}^{\mathrm{i}}} \bullet \Delta \underline{e}^{\mathrm{i}}+\frac{\partial W}{\partial \underline{e}^{\mathrm{d}}} \bullet \Delta \underline{e}^{\mathrm{d}} .
$$

Comparing (93) with (94) establishes the following result:

Proposition 13.1 In an ordinary, elastic material,

$$
\underline{t}^{\mathrm{i}}=\frac{\partial W}{\partial \underline{e}^{\mathrm{i}}}, \quad \underline{t}^{\mathrm{d}}=\frac{\partial W}{\partial \underline{e}^{\mathrm{d}}} .
$$


Applying the chain rule,

$$
\frac{\partial W}{\partial \underline{e}^{\mathrm{i}}}=\frac{\partial W}{\partial \theta} \frac{\partial \theta}{\partial \underline{e}^{\mathrm{i}}} .
$$

From the first of (90), $\theta$ is independent of $\underline{e}^{\mathrm{d}}$. Therefore, from (84), the first of (87), the first of (95), and (96),

$$
\underline{t}^{\mathrm{i}}=\frac{-3 p \underline{\omega x}}{m}=\left(\frac{3 \underline{\omega x}}{m}\right) \frac{\partial W}{\partial \theta},
$$

thus establishing the following:

Proposition 13.2 In an ordinary, elastic material,

$$
p=-\frac{\partial W}{\partial \theta}
$$

For convenience, the key relations for an ordinary, elastic material with strain energy density expressed as $W\left(\theta, \underline{e}^{\mathrm{d}}\right)$ are given here:

$$
\underline{t}=\underline{t}^{\mathrm{i}}+\underline{t}^{\mathrm{d}}, \quad \underline{t}^{\mathrm{i}}=\frac{-3 p}{m} \underline{\omega x}, \quad p=-\frac{\partial W}{\partial \theta}, \quad \underline{t}^{\mathrm{d}}=\frac{\partial W}{\partial \underline{e}^{\mathrm{d}}} .
$$

In the next three sections, materials in which $\underline{t}^{\mathrm{i}}$ depends only on $\theta$ and $\underline{t}^{\mathrm{d}}$ depends only on $\underline{e}^{\mathrm{d}}$ will be considered.

\section{Linear Peridynamic Fluids}

Definition 14.1 Suppose an ordinary, elastic peridynamic material model is given by

$$
W\left(\theta, \underline{e}^{\mathrm{d}}\right)=\frac{k \theta^{2}}{2} .
$$

where $k$ is a positive constant. Then the material is a linear peridynamic fluid.

From (99), the scalar force state and the peridynamic pressure for a linear peridynamic fluid are given by

$$
\underline{t}=\frac{3 k \theta}{m} \underline{\omega x}, \quad p=-k \theta .
$$

For this material, (87) and (95) imply that $\underline{t}^{\mathrm{i}}=\underline{t}$ and $\underline{t}^{\mathrm{d}}=\underline{0}$. Correspondence (see Section 12) of a linear peridynamic fluid with a classical linear elastic fluid at any $\mathbf{F}$ representing a small isotropic expansion implies that $k$ is the usual bulk modulus. Isotropy of this material model is closely connected with the nature of $\underline{\omega}$, as shown in the following result.

Proposition 14.1 If $\underline{\omega}$ is spherical (see Definition 3.2), then any linear peridynamic fluid is isotropic. 
Proof Let a linear peridynamic fluid be given, with $\underline{t}$ supplied by (101). Let $\mathbf{Q} \in \mathcal{Q}$. Consider an extension scalar state $\underline{e}$. From $(37,83)$, the fact that $\mathbf{Q}$ leaves vector lengths unchanged, and the assumption of spherical $\underline{\omega}$,

$$
\begin{aligned}
\hat{\theta}(\underline{e} \circ \underline{\mathbf{Q}}) & =\frac{3}{m} \int_{\mathcal{H}} \underline{\omega}_{s}\langle|\xi|\rangle|\boldsymbol{\xi}| \underline{e}\langle\underline{\mathbf{Q}}\langle\boldsymbol{\xi}\rangle\rangle d V_{\xi} \\
& =\frac{3}{m} \int_{\mathcal{H}} \underline{\omega}_{s}\langle\underline{\mathbf{Q}}\langle\boldsymbol{\xi}\rangle \mid\rangle|\underline{\mathbf{Q}}\langle\boldsymbol{\xi}\rangle| \underline{e}\langle\underline{\mathbf{Q}}\langle\boldsymbol{\xi}\rangle\rangle d V_{\boldsymbol{\xi}} \\
& =\frac{3}{m} \int_{\mathcal{H}} \underline{\omega}_{s}\langle|\zeta|\rangle|\zeta| \underline{e}\langle\zeta\rangle d V_{\zeta} \\
& =\hat{\theta}(\underline{e}) .
\end{aligned}
$$

Similarly, from this and (101), for any $\xi \in \mathcal{H}$,

$$
\begin{aligned}
\underline{\hat{t}}(\underline{e} \circ \underline{\mathbf{Q}})\langle\boldsymbol{\xi}\rangle & =\frac{3 k \hat{\theta}(\underline{e} \circ \underline{\mathbf{Q}})}{m} \underline{\omega}_{s}\langle|\xi|\rangle|\boldsymbol{\xi}| \\
& =\frac{3 k \hat{\theta}(\underline{e})}{m} \underline{\omega}_{s}\langle\underline{\mathbf{Q}}\langle\boldsymbol{\xi}\rangle \mid\rangle|\underline{\mathbf{Q}}\langle\boldsymbol{\xi}\rangle| \\
& =\underline{\hat{t}}(\underline{e})\langle\underline{\mathbf{Q}}\langle\xi\rangle\rangle \\
& =(\underline{\hat{t}}(\underline{e}) \circ \underline{\mathbf{Q}})\langle\boldsymbol{\xi}\rangle .
\end{aligned}
$$

So, the condition (54) is met, and the material is therefore isotropic.

\section{Linear Peridynamic Solids}

Definition 15.1 Suppose an ordinary, elastic peridynamic material is given by

$$
W\left(\theta, \underline{e}^{\mathrm{d}}\right)=\frac{k \theta^{2}}{2}+\frac{\alpha}{2}\left(\underline{\omega} \underline{e}^{\mathrm{d}}\right) \bullet \underline{e}^{\mathrm{d}} .
$$

where $k$ and $\alpha$ are positive constants, and where $\underline{\omega}$ is an influence function. Then the material is a linear peridynamic solid.

From (99), the force scalar state for a linear peridynamic solid is given by

$$
\underline{t}=\frac{-3 p}{m} \underline{\omega x}+\alpha \underline{\omega e}^{\mathrm{d}}, \quad p=-k \theta .
$$

The following result follows from Proposition 14.1, the decomposition (85), and the definition of the composition of two states,

Proposition 15.1 If $\underline{\omega}$ is spherical, then any linear peridynamic solid is isotropic.

It will be assumed in the remainder of this section that $\underline{\omega}$ is spherical. As with the linear peridynamic fluid, $k$ is simply the bulk modulus. To relate $\alpha$ to the shear 
modulus $\mu$ in the classical theory, consider a small homogeneous deformation with strain tensor $\epsilon_{i j}^{\mathrm{d}}$ such that $\epsilon_{k k}^{\mathrm{d}}=0$. The peridynamic material defined in (104) is required to correspond to the classical isotropic material for this deformation. As discussed in the paragraph following (85), $\theta$ equals the trace of the strain tensor for small homogeneous deformations, hence $\theta=0$ in this case. Recall from elementary kinematics that in a homogeneous deformation with deformation gradient tensor $F_{i j}=\delta_{i j}+u_{i, j}$ such that $\sqrt{u_{i, j} u_{i, j}}<<1$, the length change of a relative position vector $\xi$ is given by

$$
|\mathbf{F} \boldsymbol{\xi}|-|\boldsymbol{\xi}|=\epsilon_{i j} \xi_{i} \xi_{j} /|\boldsymbol{\xi}|, \quad \epsilon_{i j}=\left(u_{i, j}+u_{j, i}\right) / 2
$$

In the present context of peridynamic states, according to (81), this length change is $\underline{e}\langle\boldsymbol{\xi}\rangle$. Therefore, under the present assumptions, writing out the second of (85) explicitly in terms of $\boldsymbol{\xi}$, the deviatoric extension state is given by

$$
\underline{e}^{\mathrm{d}}\langle\boldsymbol{\xi}\rangle=\epsilon_{i j}^{\mathrm{d}} \xi_{i} \xi_{j} /|\xi|
$$

Now use this to compute the peridynamic elastic energy density from (104):

$$
\begin{aligned}
W & =\frac{\alpha}{2} \int_{\mathcal{H}} \underline{\omega}\langle\xi\rangle\left(\underline{e}^{\mathrm{d}}\langle\xi\rangle\right)^{2} d V_{\xi} \\
& =\frac{\alpha}{2} \int_{\mathcal{H}} \underline{\omega}\langle\boldsymbol{\xi}\rangle\left(\frac{\epsilon_{i j}^{\mathrm{d}} \xi_{i} \xi_{j}}{|\xi|}\right)\left(\frac{\epsilon_{k l}^{\mathrm{d}} \xi_{k} \xi_{l}}{|\xi|}\right) d V_{\xi} \\
& =\frac{\alpha}{2} \epsilon_{i j}^{\mathrm{d}} \epsilon_{k l}^{\mathrm{d}} \int_{\mathcal{H}} \frac{\underline{\omega}\langle\boldsymbol{\xi}\rangle}{|\boldsymbol{\xi}|^{2}} \xi_{i} \xi_{j} \xi_{k} \xi_{l} d V_{\xi} .
\end{aligned}
$$

To evaluate the integral explicitly, observe that since $\underline{\omega}$ is by assumption spherical, combinations of $\{i, j, k, l\}$ that have an odd number of any index, such as $\{1,1,3,2\}$, integrate to 0 . So, the only cases that need to be considered are those in which $i=$ $j=k=l$ or those that have two equal pairs that differ from the other pair, such as $i=k=3, j=l=1$. It therefore suffices to evaluate the following, using the spherical coordinate system $\xi_{1}=r \sin \phi \cos \theta, \xi_{2}=r \sin \phi \sin \theta, \xi_{3}=r \cos \phi$ :

$$
\int_{\mathcal{H}} \frac{\underline{\omega}_{s}\langle|\boldsymbol{\xi}|\rangle}{|\boldsymbol{\xi}|^{2}} \xi_{3}^{4} d V_{\boldsymbol{\xi}}=\int_{0}^{\delta} \int_{0}^{2 \pi} \int_{0}^{\pi} \frac{\underline{\omega}_{s}\langle r\rangle}{r^{2}}(r \cos \phi)^{4}\left(r^{2} \sin \phi d \phi d \theta d r\right)=\frac{m}{5}
$$

where, by (82), $m=4 \pi \int_{0}^{\delta} \underline{\omega}_{s}\langle r\rangle r^{4} d r$. Similarly,

$$
\int_{\mathcal{H}} \frac{\omega_{s}\langle|\boldsymbol{\xi}|\rangle}{|\boldsymbol{\xi}|^{2}} \xi_{3}^{2} \xi_{1}^{2} d V_{\xi}=\frac{m}{15}
$$

Combining (108-110), and using $\epsilon_{k k}^{d}=0$,

$$
W=\frac{\alpha m}{30} \epsilon_{i j}^{\mathrm{d}} \epsilon_{k l}^{\mathrm{d}}\left(\delta_{i j} \delta_{k l}+\delta_{i k} \delta_{j l}+\delta_{i l} \delta_{j k}\right)=\frac{\alpha m}{15} \epsilon_{i j}^{\mathrm{d}} \epsilon_{i j}^{\mathrm{d}} .
$$

It is now required that the isotropic linear peridynamic solid correspond to the classical isotropic linear elastic solid for all deformations of this form, i.e., with no dilatation. This requirement means that the last expression in (111) must agree with 
the well-known classical result for strain energy density, $\Omega=\mu \epsilon_{i j}^{\mathrm{d}} \epsilon_{i j}^{\mathrm{d}}$, where $\mu$ is the shear modulus. Thus,

$$
\alpha=\frac{15 \mu}{m} .
$$

Combining this result with (105) leads to an expression for the force state in a peridynamic linear isotropic solid in which only the classical elastic moduli appear as parameters:

$$
\underline{t}=\frac{-3 p}{m} \underline{\omega x}+\frac{15 \mu}{m} \underline{\omega e}^{\mathrm{d}}, \quad p=-k \theta .
$$

The structure of the peridynamic constitutive model in (113) is evidently similar to the classical model, $\boldsymbol{\sigma}=-p \mathbf{1}+2 \mu \epsilon^{\mathrm{d}}, p=-k \theta$.

\section{Peridynamic Elastic-Plastic Materials}

The aim of this section is to construct a constitutive model in the state based peridynamic theory analogous to an elastic-plastic flow model in the classical theory. This model will include the decoupling of plastic flow from volume changes and pressure, an idealization called plastic incompressibility, which describes the permanent deformation of many metals and some other non-porous materials with good accuracy. The strategy is to define a yield surface in $\mathcal{S}^{\mathrm{d}}$, the space of co-deviatoric force states:

$$
\mathcal{S}^{\mathrm{d}}=\left\{\underline{t}^{\mathrm{d}} \in \mathcal{S} \mid \underline{t}^{\mathrm{d}} \bullet \underline{x}=0\right\} .
$$

Observe that $\mathcal{S}^{\mathrm{d}}$ is a subspace of $\mathcal{S}$. At this point, it is necessary to introduce a modified definition of Frechet derivative $\nabla^{\mathrm{d}} \psi$ (see Definition 5.1) appropriate for functions on this subspace.

Definition 16.1 Let $\psi: \mathcal{S}^{\mathrm{d}} \rightarrow \mathbb{R}$ be a scalar-valued function on $\mathcal{S}^{\mathrm{d}}$. Suppose there exists a scalar state-valued function denoted $\nabla^{\mathrm{d}} \psi: \mathcal{S}^{\mathrm{d}} \rightarrow \mathcal{S}$ such that both of the following hold:

(1) For any $\underline{a}^{\mathrm{d}} \in \mathcal{S}^{\mathrm{d}}$,

$$
\hat{\theta}\left(\nabla^{\mathrm{d}} \psi\left(\underline{a}^{\mathrm{d}}\right)\right)=0
$$

(2) For any $\underline{a}^{\mathrm{d}} \in \mathcal{S}^{\mathrm{d}}$ and any $\Delta \underline{a}^{\mathrm{d}} \in \mathcal{S}^{\mathrm{d}}$,

$$
\psi\left(\underline{a}^{\mathrm{d}}+\Delta \underline{a}^{\mathrm{d}}\right)=\psi\left(\underline{a}^{\mathrm{d}}\right)+\nabla^{\mathrm{d}} \psi\left(\underline{a}^{\mathrm{d}}\right) \bullet \Delta \underline{a}^{\mathrm{d}}+o\left(\left\|\Delta \underline{a}^{\mathrm{d}}\right\|\right),
$$

where $\hat{\theta}$ is defined in (83). Then $\nabla^{\mathrm{d}} \psi$ is called the constrained Frechet derivative of $\psi$ on $\mathcal{S}^{\mathrm{d}}$. (Note that in general $\nabla^{\mathrm{d}} \psi \notin \mathcal{S}^{\mathrm{d}}$.)

To compute $\nabla^{\mathrm{d}} \psi$, let $\psi$ be a scalar-valued function $\mathcal{S}$, differentiable in the (unconstrained) sense of Definition 5.1. Let

$$
\hat{t^{\mathrm{d}}}(\underline{t})=\underline{t}-\frac{\underline{t} \bullet \underline{x}}{m} \underline{\omega x} \quad \forall \underline{t} \in \mathcal{S} .
$$


Now consider the quantity $g=\partial \psi\left(\underline{t}^{\mathrm{d}}(\underline{t})\right) / \partial \underline{t}$ [see (25) for the meaning of this notation; it is not the same as $\nabla \psi(\underline{t}(\underline{\mathrm{d}}))]$. From (117), applying the chain rule leads to

$$
\underline{g}=\nabla \psi-\frac{\nabla \psi \bullet \underline{(\omega x)}}{m} \underline{x} .
$$

From $(82,83)$, and $(118)$, it follows that $\hat{\theta}(g)=0$. Also, for any $\Delta \underline{a}^{\mathrm{d}} \in \mathcal{S}^{\mathrm{d}}$, from (114) and (118), we have $g \bullet \Delta \underline{a}^{\mathrm{d}}=\nabla \psi \bullet \Delta \underline{a}^{\mathrm{d}}=\Delta \psi$. Thus, $g$ meets the requirements of Definition 16.1, and the following lemma is established:

Lemma 16.1 Let $\psi: \mathcal{S} \rightarrow \mathbb{R}$ be a differentiable nonnegative scalar-valued function of a scalar state. Then the constrained Frechet derivative of $\psi$ on $\mathcal{S}^{\mathrm{d}}$ exists and is given by

$$
\nabla^{\mathrm{d}} \psi=\nabla \psi-\frac{\nabla \psi \bullet(\underline{\omega x})}{m} \underline{x} .
$$

Note that this lemma assumes that $\psi$ is defined on (and is differentiable on) $\mathcal{S}$, not just $\mathcal{S}^{\mathrm{d}}$. This assumption is necessary because $\nabla \psi$ is involved. For a $\psi$ that is undefined on $\mathcal{S}-\mathcal{S}^{\mathrm{d}}$, some means other than (119) would need to be found for evaluating $\nabla^{\mathrm{d}} \psi$. However, this is not a practical difficulty for purposes of the following discussion.

The motivation for requiring (115) as the appropriate constraint on $\nabla^{\mathrm{d}} \psi$ is found in the third of (90), which states that co-deviatoric force states are orthogonal to isotropic extension states. Thus, if one is seeking a state $\nabla^{\mathrm{d}} \psi$ that is orthogonal to $\mathcal{S}^{\mathrm{d}}$, this required state is deviatoric, hence $\hat{\theta}\left(\nabla^{\mathrm{d}} \psi\right)=0$. Now the idea of a constrained derivative will be used to construct elastic and plastic parts of an incremental extension state.

Definition 16.2 Let $\psi$ be a differentiable scalar-valued function on $\mathcal{S}$ such that $\psi(\underline{0})=0$. Let $\psi_{0}>0$ be a fixed scalar. Define

$$
\mathrm{Y}=\left\{\underline{t}^{\mathrm{d}} \in \mathcal{S}^{\mathrm{d}} \mid \psi\left(\underline{t}^{\mathrm{d}}\right)=\psi_{0}\right\}
$$

Then $\mathrm{Y}$ is called a yield surface in $\mathcal{S}^{\mathrm{d}}$.

Since $\mathcal{S}^{\mathrm{d}}$ is an infinite-dimensional space, the peridynamic yield surface is a somewhat more abstract object than a yield surface in the classical theory of plasticity; yet the basic idea is the same (Fig. 4). Now a constitutive model for plasticity will be proposed. The material to be described is not simple (see Definition 8.2) because it inherently involves history dependence.

Definition 16.3 Let $\psi$ be a differentiable scalar-valued function on $\mathcal{S}$. Let $\psi_{0}$ be a fixed scalar, and let $Y$ be the corresponding yield surface in $\mathcal{S}^{\mathrm{d}}$. Define a constitutive model for the rate $\underline{\dot{t}}$ expressed in terms of the rate $\underline{\dot{e}}$ as follows:

$$
\begin{gathered}
\dot{\underline{t}}=\underline{\dot{t}}^{\mathrm{i}}+\underline{\dot{t}}^{\mathrm{d}}, \\
\underline{t}^{\mathrm{i}}=\frac{3 k \dot{\theta}}{m} \underline{\omega x}, \quad \dot{\theta}=\hat{\theta}(\underline{\dot{e}}), \\
\underline{\dot{t}}^{\mathrm{d}}=\alpha \underline{\underline{\omega}}^{\mathrm{de}}, \quad \underline{\dot{e}}^{\mathrm{de}}=\underline{\dot{e}}^{\mathrm{d}}-\underline{\dot{e}}^{\mathrm{dp}}, \quad \underline{\dot{e}}^{\mathrm{dp}}=\lambda \nabla^{\mathrm{d}} \psi,
\end{gathered}
$$


Fig. 4 Schematic of a yield surface for a peridynamic plastic material. The surface exists in an infinite dimensional space

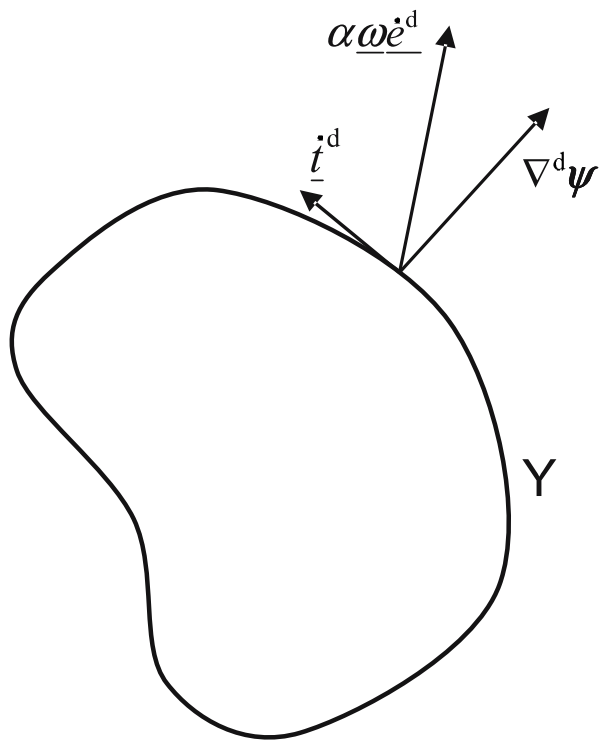

$\lambda= \begin{cases}0 & \text { if } \psi\left(\underline{t}^{\mathrm{d}}\right)<\psi_{0} \text { or }\left(\underline{\omega \dot{e}}^{\mathrm{d}}\right) \bullet \nabla^{\mathrm{d}} \psi<0 \\ \lambda^{\mathrm{p}} & \text { otherwise }\end{cases}$

$$
\lambda^{\mathrm{p}}=\frac{\left(\underline{\omega}^{\mathrm{d}}\right) \bullet \nabla^{\mathrm{d}} \psi}{\left(\underline{\omega}^{\mathrm{d}} \psi\right) \bullet \nabla^{\mathrm{d}} \psi}
$$

where $\nabla^{\mathrm{d}} \psi=\nabla^{\mathrm{d}} \psi\left(\underline{t}^{\mathrm{d}}\right)$, and $\alpha$ is a positive constant that has the same meaning and value as in (104). The material described by (121-125) is called the peridynamic elastic-plastic solid.

To ensure that the denominator in (125) is nonzero, an additional assumption is now made:

$$
\left(\underline{\omega} \nabla^{\mathrm{d}} \psi\right) \bullet \nabla^{\mathrm{d}} \psi>0 .
$$

In view of the third of (123), and thinking of $\alpha \underline{\omega}^{\mathrm{dp}}$ as the plastic part of $\underline{\dot{d}}^{\mathrm{d}}$, this assumption may be interpreted as a material stability requirement. In this interpretation, the requirement is that the plastic work provided by an increment in $\underline{e}^{\mathrm{dp}}$ acting through the resulting increment in the force state is positive. This is remarkably similar to the standard stability requirement due to Drucker in the classical theory of plasticity [19]. A sufficient condition for (126) is that $\underline{\omega}$ be strictly positive on $\mathcal{H}$. The scalar states $\underline{\dot{e}}^{\mathrm{de}}$ and $\underline{\dot{e}}^{\mathrm{dp}}$ are called the elastic and plastic parts of the deviatoric extension rate, respectively. If $\psi\left(t^{\mathrm{d}}\right)<\psi_{0}$, then $\underline{t}^{\mathrm{d}}$ is said to be inside the yield surface $\mathrm{Y}$; if $\psi\left(\underline{t}^{\mathrm{d}}\right)=\psi_{0}$, then it is said to be on the yield surface.

Since the peridynamic elastic-plastic solid is defined in terms of rates, it is necessary to include some initial condition, which for the sake of definiteness will be assumed to be $\underline{t}=\underline{0}$ at $t=0$. To show that $\underline{t}^{\mathrm{d}}$ cannot venture outside the yield 
surface, it is easily confirmed using (121-125) that if $\underline{t}^{\mathrm{d}}$ is on $\mathrm{Y}$, then $\underline{t}^{\mathrm{d}} \bullet \nabla^{\mathrm{d}} \psi=0$. From (116), this imples $\dot{\psi}=0$.

All that remains is to confirm that the evolution of $t$ implied by the definition of the peridynamic elastic-plastic solid leaves the peridynamic pressure unchanged. To confirm this, use $(83,86,119,121-125)$, and the identity $\underline{x} \bullet\left(\underline{\omega e^{\mathrm{d}}}\right)=0$ to compute

$$
\begin{aligned}
\dot{p} & =-\frac{\dot{t} \bullet \underline{x}}{3}=-\frac{\left(\underline{\dot{t}}^{\mathrm{i}}+\underline{\dot{t}}^{\mathrm{d}}\right) \bullet \underline{x}}{3} \\
& =-k \dot{\theta}-(\underline{x} / 3) \bullet\left\{\alpha \underline{\omega \dot{e}^{\mathrm{d}}}-\lambda \underline{\omega} \nabla^{\mathrm{d}} \psi\right\} \\
& =-k \dot{\theta}+(\lambda \underline{x} / 3) \bullet\{\lambda \underline{\omega}[\nabla \psi-(\nabla \psi \bullet(\underline{\omega x} / m)) \underline{x}]\} \\
& =-k \dot{\theta}+(\lambda / 3)\{\underline{x} \bullet(\underline{\omega} \nabla \psi)-(\nabla \psi \bullet(\underline{\omega x} / m)) \underline{x} \bullet(\underline{\omega x})\} \\
& =-k \dot{\theta}+(\lambda / 3)\{\underline{x} \bullet(\underline{\omega} \nabla \psi)-\nabla \psi \bullet(\underline{\omega x})\} \\
& =-k \dot{\theta} .
\end{aligned}
$$

The conclusion is that $p$ depends only on $\theta$ and is unaffected by the plastic part of the extension. Recall from (115) and the third of (123) that the plastic part of the extension has no dilatation associated with it. These two observations show that the material described by (121-125) exhibits plastic incompressibility, i.e., the pressuredilatation response is decoupled from the plastic response.

Example Suppose $\psi\left(\underline{t}^{\mathrm{d}}\right)=\left\|\underline{t}^{\mathrm{d}}\right\|^{2} / 2$ and $\underline{\omega} \equiv 1$. Then, for force states on the yield surface $\psi=\psi_{0},(121-125)$ result in

$$
\begin{gathered}
\underline{\dot{e}}^{\mathrm{dp}}=\frac{\underline{\dot{e}}^{\mathrm{d}} \bullet \underline{t}^{\mathrm{d}}}{\left\|\underline{t}^{\mathrm{d}}\right\|^{2}} \underline{t}^{\mathrm{d}}, \\
\underline{t}^{\mathrm{d}}=\alpha\left(\underline{\dot{e}}^{\mathrm{d}}-\frac{\underline{\dot{e}}^{\mathrm{d}} \bullet \underline{t}^{\mathrm{d}}}{\left\|\underline{t}^{\mathrm{d}}\right\|^{2}} \underline{t}^{\mathrm{d}}\right),
\end{gathered}
$$

from which it is immediate that $\underline{t}^{\mathrm{d}} \bullet \underline{t}^{\mathrm{d}}=0$, hence $\partial\left\|\underline{t}^{\mathrm{d}}\right\| / \partial t=0$.

\section{Example of a Nonordinary Material}

Nonordinary materials are interesting because they represent a substantial break with the classical theory of continuum mechanics. Recall from Definition 8.4 that such materials have, for some $\boldsymbol{\xi} \in \mathcal{H}$, a force density vector $\underline{\mathbf{T}}\langle\boldsymbol{\xi}\rangle$ that is not parallel to the deformed image of $\xi$. To demonstrate that such materials can have reasonable properties, consider the force state given by

$$
\underline{\mathbf{T}}\langle\boldsymbol{\xi}\rangle=\frac{\underline{\mathbf{Y}}\langle\boldsymbol{\xi}\rangle \times \underline{\mathbf{H}}(\underline{\mathbf{Y}})\langle\boldsymbol{\xi}\rangle}{|\underline{\mathbf{Y}}\langle\boldsymbol{\xi}\rangle|^{2}} \quad \forall \xi \in \mathcal{H}
$$

where $\underline{\mathbf{H}}(\cdot): \mathcal{V} \rightarrow \mathcal{V}$ is a vector state valued function such that

$$
\underline{\mathbf{H}}\langle-\boldsymbol{\xi}\rangle=-\underline{\mathbf{H}}\langle\xi\rangle \quad \text { and } \quad \underline{\mathbf{H}}(\underline{\mathbf{Y}})\langle\xi\rangle \cdot \underline{\mathbf{Y}}\langle\xi\rangle=\mathbf{0} \quad \forall \xi \in \mathcal{H} .
$$




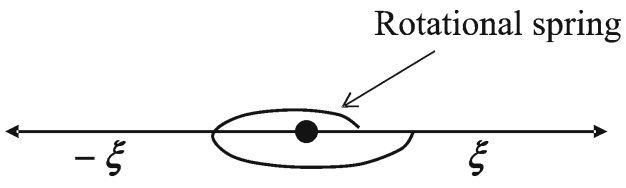

Reference configuration

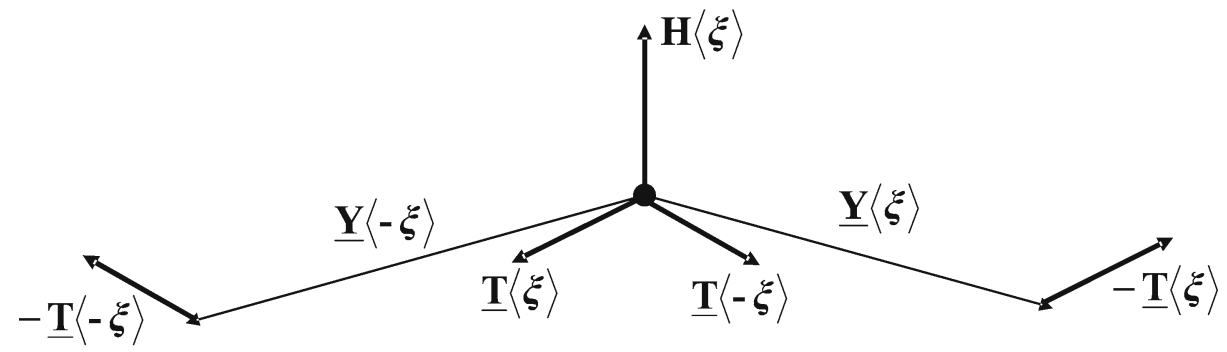

Deformed configuration

Fig. 5 Example of a non-ordinary material. Each pair of opposite bonds is connected by a rotational spring that resists the two bonds' relative rotation. The force couples $\pm \mathbf{T}\langle\boldsymbol{\xi}\rangle$ and $\pm \mathbf{T}\langle-\boldsymbol{\xi}\rangle$ are determined by the constitutive model such that their sum produces zero moment, hence this model satisfies balance of angular momentum

The interpretation of (130) is that each vector $\mathbf{H}\langle\boldsymbol{\xi}\rangle$ represents minus the moment that the force $\underline{\mathbf{T}}\langle\boldsymbol{\xi}\rangle$ exerts through the bond (Fig. 5). Suppose $\mathbf{H}$ depends only on the relative angle change of the vectors $\xi$ and $-\xi$ under deformation, e.g.,

$$
\underline{\mathbf{H}}\langle\boldsymbol{\xi}\rangle=\alpha \underline{\mathbf{M}}\langle\boldsymbol{\xi}\rangle \times \underline{\mathbf{M}}\langle-\boldsymbol{\xi}\rangle \quad \forall \xi \in \mathcal{H},
$$

where $\alpha$ is a constant. Then this material model can be thought of as a being composed of rotational springs that resist changes in relative angle between vectors $\xi$ and $-\xi$. The requirement for balance of angular momentum (34) is satisfied for this material because the sum of the moments through opposite bonds $\boldsymbol{\xi}$ and $-\boldsymbol{\xi}$ vanishes. To see this combine (130) and (131), and use the "BAC-CAB" vector identity, $\mathbf{a} \times(\mathbf{b} \times \mathbf{c})=\mathbf{b}(\mathbf{a} \cdot \mathbf{c})-\mathbf{c}(\mathbf{a} \cdot \mathbf{b})$, to confirm that

$$
\underline{\mathbf{Y}}\langle\boldsymbol{\xi}) \times \underline{\mathbf{T}}\langle\boldsymbol{\xi}\rangle+\underline{\mathbf{Y}}\langle-\boldsymbol{\xi}\rangle \times \underline{\mathbf{T}}\langle-\boldsymbol{\xi}\rangle=\mathbf{0} . \quad \forall \boldsymbol{\xi} \in \mathcal{H} .
$$

Thus, (41) holds for this material, and therefore balance of angular momentum is satisfied.

\section{Adaptation of Classical Material Models}

Suppose a hyperelastic material (in the sense of classical elasticity theory) has a given strain energy density function $\Omega(\mathbf{F})$, where $\mathbf{F}$ is the deformation gradient tensor. Can this material model be applied in the state-based peridynamic framework? 
The deformation vector state $\underline{\mathbf{Y}}$ represents a much more general way of describing how a body deforms locally than the classical idea of a deformation gradient tensor F. However, given $\underline{\mathbf{Y}}$, there is nothing to prevent us from deriving a corresponding deformation gradient tensor, which will be called $\overline{\mathbf{F}}$, using reduction as defined in (19):

$$
\overline{\mathbf{F}}(\underline{\mathbf{Y}})=\mathcal{R}\{\underline{\mathbf{Y}}\}=(\underline{\mathbf{Y}} * \underline{\mathbf{X}}) \mathbf{K}^{-1} .
$$

Then a peridynamic elastic material can be defined directly from the given classical model:

$$
W(\underline{\mathbf{Y}})=\Omega(\overline{\mathbf{F}}(\underline{\mathbf{Y}})),
$$

and the resulting force vector state can be evaluated from (61).

To carry this out, note that from $(16,17,19)$, and (134), the Frechet derivative of $\overline{\mathbf{F}}$ may be evaluated using components by considering an incremental change in deformation state $\underline{\mathbf{Y}}$ :

$$
\begin{aligned}
\overline{\mathbf{F}}_{i j}(\underline{\mathbf{Y}}+\Delta \underline{\mathbf{Y}}) & =\int_{\mathcal{H}} \underline{\omega}\langle\boldsymbol{\xi}\rangle\left(\underline{y}_{i}\langle\boldsymbol{\xi}\rangle+\Delta \underline{y}_{i}\langle\boldsymbol{\xi}\rangle\right) \xi_{p} K_{p j}^{-1} d V_{\boldsymbol{\xi}} \\
& =\overline{\mathbf{F}}_{i j}(\underline{\mathbf{Y}})+\int_{\mathcal{H}} \underline{\omega}\langle\boldsymbol{\xi}\rangle \Delta \underline{y}_{i}\langle\boldsymbol{\xi}\rangle \xi_{p} K_{p j}^{-1} d V_{\boldsymbol{\xi}} \\
& =\overline{\mathbf{F}}_{i j}(\underline{\mathbf{Y}})+\int_{\mathcal{H}} \underline{\omega}\langle\boldsymbol{\xi}\rangle \Delta \underline{y}_{k}\langle\boldsymbol{\xi}\rangle \delta_{i k} \xi_{p} K_{p j}^{-1} d V_{\boldsymbol{\xi}} \\
& =\overline{\mathbf{F}}_{i j}(\underline{\mathbf{Y}})+\left(\delta_{i k} \underline{\omega}_{p j}^{-1} \underline{x}_{p}\right) \bullet \Delta \underline{y}_{k},
\end{aligned}
$$

hence, from (23),

$$
\nabla \bar{F}_{i j k}(\underline{\mathbf{Y}})=\delta_{i k} \underline{\omega}_{p j}^{-1} \underline{x}_{p},
$$

or equivalently,

$$
\nabla \bar{F}_{i j k}(\underline{\mathbf{Y}})\langle\boldsymbol{\xi}\rangle=\delta_{i k} \underline{\omega}\langle\boldsymbol{\xi}\rangle K_{p j}^{-1} \xi_{p} .
$$

( $\nabla \overline{\mathbf{F}}$ is a slightly odd bird: a state of order 3.) Returning temporarily to the classical theory, the Piola stress tensor $\sigma$ is related to the strain energy density through the tensor gradient,

$$
\sigma_{i j}(\overline{\mathbf{F}})=\frac{\partial \Omega}{\partial F_{i j}}(\overline{\mathbf{F}}) .
$$

Now compute the incremental change in $W$ due to an incremental change in $\underline{\mathbf{Y}}$, using (137) and (139),

$$
\begin{aligned}
\Delta W & =\frac{\partial \Omega}{\partial \bar{F}_{i j}} \Delta \bar{F}_{i j} \\
& =\frac{\partial \Omega}{\partial F_{i j}} \nabla \bar{F}_{i j k} \bullet \Delta \underline{y}_{k} \\
& =\left(\sigma_{i j} \delta_{i k} \underline{\omega}_{p j}^{-1} \underline{x}_{p}\right) \bullet \Delta \underline{y}_{k} \\
& =\left(\sigma_{k j} \underline{\omega} K_{p j}^{-1} \underline{x}_{p}\right) \bullet \Delta \underline{y}_{k} .
\end{aligned}
$$


Comparing (140) with (61) and recalling that $\mathbf{K}$ is symmetric leads to the conclusion

$$
\underline{T}_{i}=\sigma_{i j} \underline{\omega}_{j p}^{-1} \underline{x}_{p} .
$$

Equivalently, using (15),

$$
\underline{\mathbf{T}}\langle\boldsymbol{\xi}\rangle=\underline{\omega}\langle\boldsymbol{\xi}\rangle \boldsymbol{\sigma} \mathbf{K}^{-1} \boldsymbol{\xi} \quad \text { or } \quad \underline{\mathbf{T}}=\underline{\omega \mathcal{E}}\left\{\boldsymbol{\sigma} \mathbf{K}^{-1}\right\} .
$$

Equations $(134,139)$, and (142) together form the peridynamic constitutive model based on a classical constitutive model. There is one remaining step, which is to confirm that the peridynamic model obeys the balance of angular momentum (see Proposition 8.1). To confirm this, first recall the relation between $\sigma$ and the Cauchy stress tensor $\boldsymbol{\tau}$ :

$$
\sigma=J \tau \mathbf{F}^{-T}, \quad J=\operatorname{det} \mathbf{F} .
$$

Let $\epsilon_{i j k}$ denote the alternator tensor components. Now calculate the net moment on the force state $\mathbf{T}$ using $(134,142,143)$, and the symmetry of $\boldsymbol{\tau}$ and $\mathbf{K}$ :

$$
\begin{aligned}
\left(\int_{\mathcal{H}} \underline{\mathbf{Y}}\langle\boldsymbol{\xi}\rangle \times \underline{\mathbf{T}}\langle\boldsymbol{\xi}\rangle d V_{\boldsymbol{\xi}}\right)_{i} & =\epsilon_{i j k} \int_{\mathcal{H}} \underline{y}_{j}\langle\boldsymbol{\xi}\rangle\left(\underline{\omega}\langle\boldsymbol{\xi}\rangle \sigma_{k p} K_{p r}^{-1} \xi_{r}\right) d V_{\boldsymbol{\xi}} \\
& =\epsilon_{i j k} \int_{\mathcal{H}} \underline{y}_{j}\langle\boldsymbol{\xi}\rangle\left(\underline{\omega}\langle\boldsymbol{\xi}\rangle\left(J \tau_{k q} F_{p q}^{-1}\right) K_{p r}^{-1} \xi_{r}\right) d V_{\boldsymbol{\xi}} \\
& =\epsilon_{i j k}\left(J \tau_{k q} F_{p q}^{-1}\right) K_{p r}^{-1}\left(\int_{\mathcal{H}} \underline{\omega}\langle\boldsymbol{\xi}\rangle \underline{y}_{j}\langle\boldsymbol{\xi}\rangle \xi_{r} d V_{\boldsymbol{\xi}}\right) \\
& =\epsilon_{i j k}\left(J \tau_{k q} F_{p q}^{-1}\right) K_{p r}^{-1}\left(F_{j s} K_{s r}\right) \\
& =J \epsilon_{i j k} \tau_{k q}\left(F_{p q}^{-1} F_{j s}\right)\left(K_{p r}^{-1} K_{s r}\right) \\
& =J \epsilon_{i j k} \tau_{k q}\left(F_{p q}^{-1} F_{j s}\right) \delta_{p s} \\
& =J \epsilon_{i j k} \tau_{k q} F_{p q}^{-1} F_{j p} \\
& =J \epsilon_{i j k} \tau_{k q} \delta_{j q} \\
& =J \epsilon_{i j k} \tau_{k j} \\
& =0
\end{aligned}
$$

so (41) holds. This proves the perhaps suprising result that $\underline{\mathbf{T}}$ obtained from (142) satisfies the balance of angular momentum, and that this holds for any choice of $\underline{\omega}$ that satisfies the conditions in Definition 3.2, not just spherical functions.

This completes the process of applying a classical material model in the state based peridynamic framework. The process is summarized as follows:

- The approximate deformation gradient $\overline{\mathbf{F}}$ is determined by (134).

- The corresponding Piola stress tensor $\sigma$ is found from the constitutive model (139), or from some other material model. (Equivalently, the Cauchy stress tensor $\tau$ may be determined from a constitutive model and then converted to $\sigma$ using (143).)

- The Piola stress tensor is converted to a force state $\underline{\mathbf{T}}$ using (142). 
Recent computational results implementing the method just described have demonstrated good agreement between the peridynamic and conventional methods applied to the stretching of an elastic-plastic bar in uniaxial stress [20].

Interfaces, free surfaces, and mixtures all may be treated in the same way using suitable choices of the influence function $\underline{\omega}$. For example, if $\mathbf{x}$ is located near a free boundary, $\underline{\omega}$ would be chosen such that it vanishes at all points in the horizon that are outside the body. Similarly, if $\mathbf{x}$ is near an interface between two materials denoted (1) and (2), two separate influence functions $\underline{\omega}^{(1)}$ and $\underline{\omega}^{(2)}$ can be defined such that each vanishes at points $\mathbf{x}+\boldsymbol{\xi}$ outside its respective material. These two influence functions, when applied to the deformation state $\underline{\mathbf{Y}}$ using (134), result in separate deformation gradient tensors for the two materials, $\overline{\mathbf{F}}^{(1)}$ and $\overline{\mathbf{F}}^{(2)}$. These yield two separate stress tensors $\sigma^{(1)}$ and $\sigma^{(2)}$ from the respective constitutive models. Finally, the two force states corresponding to these stress tensors are combined to give

$$
\underline{\mathbf{T}}=\left(\underline{\omega} \underline{\mathcal{E}}\left\{\boldsymbol{\sigma} \mathbf{K}^{-1}\right\}\right)^{(1)}+\left(\underline{\omega} \mathcal{E}\left\{\boldsymbol{\sigma} \mathbf{K}^{-1}\right\}\right)^{(2)} .
$$

There is no assumption regarding smoothness or regularity of the interface other than the rather weak conditions on the influence functions in Definition 3.2. So, in principle, complex interfaces and mixtures could be modeled with this approach.

Finally, the framework outlined above provides a means to include damage in a material model for the force state. One way of doing this is to define a scalar state $\mu \in \mathcal{S}$ that takes on a value of 1 or 0 to indicate undamaged or damaged bonds, respectively, connecting $\mathbf{x}$ to $\mathbf{x}+\xi$. The criterion for bond breakage is flexible. One possible criterion is that $\mu\langle\boldsymbol{\xi}\rangle$ changes irreversibly from 1 to 0 whenever the corresponding bond extension $\underline{\underline{e}}\langle\boldsymbol{\xi}\rangle$ exceeds some prescribed critical value $e^{*}$ as the bond deforms over time. The influence function $\underline{\omega}$ is chosen such that it excludes damaged bonds, i.e.,

$$
\underline{\mu}\langle\boldsymbol{\xi}\rangle=0 \Longrightarrow \underline{\omega}\langle\boldsymbol{\xi}\rangle=0 .
$$

This ensures that damaged bonds are excluded from the calculation of $\overline{\mathbf{F}}$ and that the resulting force state $\underline{\mathbf{T}}$ takes on a value of $\underline{\mathbf{0}}$ at damaged bonds.

\section{Summary}

This paper has presented a generalization of the bond-based peridynamic theory proposed in [1]. This generalization achieves several significant improvements in the original theory:

- It models materials with Poisson ratios other than $1 / 4$, including fluids.

- It allows constitutive models from the classical theory of elasticity to be incorporated directly within the peridynamic approach.

- It incorporates features of material response, including damage evolution, that involve collective behavior of all the points with which a given $\mathbf{x}$ interacts. This is in contrast to the assumption in the original theory that interaction between each pair of particles is independent of all the others.

- It allows for plasticity and plastic incompressibility.

空 Springer 
- It allows for force vectors within bonds that are not necessarily directed parallel to the deformed bond direction.

These improvements do not diminish the major advantage of the original bond-based theory, which is the ability to apply the fundamental equations directly on a surface of discontinuity.

Acknowledgements Sandia is a multiprogram laboratory operated by Sandia Corporation, a Lockheed Martin Company, for the US Department of Energy's National Nuclear Security Administration under contract DE-AC04-94AL85000. This work was partially supported by The Boeing Company and by the Computer Science Research Foundation at Sandia National Laboratories. Helpful discussions with Dr. Thomas L. Warren and Professor Florin Bobaru are gratefully acknowledged.

\section{References}

1. Silling, S.A.: Reformulation of elasticity theory for discontinuities and long-range forces. J. Mech. Phys. Solids 48, 175-209 (2000)

2. Silling, S.A., Bobaru, F.: Peridynamic modeling of membranes and fibers. Int. J. Non-linear Mech. 40, 395-409 (2005)

3. Kunin, I.A.: Elastic Media with Microstructure I: One-dimensional Models, pp. 27. Springer, Berlin (1982)

4. Rogula, D.: Nonlocal Theory of Material Media, pp. 137-149 and 243-278. Springer, Berlin (1982)

5. Silling, S.A., Zimmermann, M., Abeyaratne, R.: Deformation of a peridynamic bar. J. Elast. 73, 173-190 (2003)

6. Weckner, O., Abeyaratne, R.: The effect of long-range forces on the dynamics of a bar. J. Mech. Phys. Solids 53, 705-728 (2005)

7. Dayal, K., Bhattacharya, K.: Kinetics of phase transformations in the peridynamic formulation of solid mechanics. J. Mech. Phys. Solids 54, 1811-1842 (2006)

8. Gerstle, W., Sau, N.: Peridynamic modeling of concrete structures. In: Li, Leung, Willam, Billington (eds.) Proceedings of the 5th International Conference on Fracture Mechanics of Concrete Structures, Ia-FRAMCOS, vol. 2, pp. 949-956 (2004)

9. Gerstle, W., Sau, N., Silling, S.: Peridynamic modeling of plain and reinforced concrete structures. In: 18th International Conference on Structural Mechanics in Reactor Technology (SMiRT 18), Beijing, China, SMiRT18-B01-2 (2005)

10. Gerstle, W., Sau, N., Silling, S.: Peridynamic modeling of concrete structures. Nucl. Eng. Des. 237, 1250-1258 (2007)

11. Silling, S.A., Askari, E.: Peridynamic modeling of impact damage. In: Moody, F.J. (ed.) Problems Involving Thermal-Hydraulics, Liquid Sloshing, and Extreme Loads on Structures, PVP-vol. 489, pp. 197-205. American Society of Mechanical Engineers, New York (2004)

12. Askari, A., Xu, J., Silling, S.: Peridynamic analysis of damage and failure in composites. In: 44th AIAA Aerospace Sciences Meeting and Exhibit, Reno, Nevada, AIAA-2006-88 (2006)

13. Kilic, B., Madenci, E., Ambur, D.R.: Analysis of brazed single-lap joints using the peridynamics theory. In: 47th AIAA/ASME/ASCE/AHS/ASC Structures, Structural Dynamics, and Materials Conference, Newport, Rhode Island, AIAA-2006-2267 (2006)

14. Bobaru, F., Silling, S.A.: Peridynamic 3D models of nanofiber networks and carbon nanotubereinforced composites. In: Materials Processing and Design: Modeling, Simulation, and Applications - NUMIFORM 2004 - American Institute of Physics Conference Proceedings, vol. 712, pp. $1565-1570$ (2004)

15. Silling, S.A.: Dynamic fracture modeling with a meshfree peridynamic code. In: Bathe, K.J. (ed.) Computational Fluid and Solid Mechanics 2003, pp. 641-644. Elsevier, Amsterdam (2003)

16. Silling, S.A., Askari, E.: A meshfree method based on the peridynamic model of solid mechanics. Comput. Struct. 83, 1526-1535 (2005)

17. Weckner, O., Emmrich, E.: Numerical simulation of the dynamics of a nonlocal, inhomogeneous, infinite bar. J. Comput. Appl. Mech. 6, 311-319 (2005) 
18. Knowles, J.K.: Linear Vector Spaces and Cartesian Tensors, pp. 1-9. Oxford University Press, New York (1998)

19. Malvern, L.E.: Introduction to the Mechanics of a Continuous Medium. Englewood Cliffs, p. 359. Prentice-Hall, New Jersey (1969)

20. Warren, T.L., Silling, S.A., Askari, E., Weckner, O., Epton, M., Xu, J.: A non-ordinary sate based peridynamic method using an isotropic elastic-plastic constitutive model, (2007) (in preparation) 